\title{
Surgical Robotics - Visually Autonomous Cauterization System (VACS) Conceptual Design by
}

\author{
Nicholas A. Spooner, B.Eng. \\ Carleton University
}

\author{
A thesis submitted to the \\ the Faculty of Graduate Studies and Research \\ in partial fulfillment of the requirements \\ for the degree of

\section{Master of Applied Science} \\ Ottawa-Carleton Institutc for \\ Mechanical and Aerospace Engineering
}

\author{
Department of Mechanical and Aerospace Engineering \\ Carleton University, \\ Division of Cardiovascular Devices \\ University of Ottawa Heart Institute \\ Ottawa, Ontario, Canada. \\ November 2006
}

Copyright (C) Division of Cardiovascular Devices, Nicholas A. Spooner, B.Eng., 2006 


$\begin{array}{ll}\begin{array}{l}\text { Library and } \\ \text { Archives Canada }\end{array} & \begin{array}{l}\text { Bibliothèque et } \\ \text { Archives Canada }\end{array} \\ \begin{array}{l}\text { Published Heritage } \\ \text { Branch }\end{array} & \begin{array}{l}\text { Direction du } \\ \text { Patrimoine de l'édition }\end{array} \\ \begin{array}{l}\text { 395 Wellington Street } \\ \text { Ottawa ON K1A ON4 }\end{array} & \begin{array}{l}\text { 395, rue Wellington } \\ \text { Ottawa ON K1A ON4 } \\ \text { Canada }\end{array}\end{array}$

Your file Votre référence ISBN: 978-0-494-23349-8 Our file Notre référence ISBN: $978-0-494-23349-8$

NOTICE:

The author has granted a nonexclusive license allowing Library and Archives Canada to reproduce, publish, archive, preserve, conserve, communicate to the public by telecommunication or on the Internet, loan, distribute and sell theses worldwide, for commercial or noncommercial purposes, in microform, paper, electronic and/or any other formats.

The author retains copyright ownership and moral rights in this thesis. Neither the thesis nor substantial extracts from it may be printed or otherwise reproduced without the author's permission.
AVIS:

L'auteur a accordé une licence non exclusive permettant à la Bibliothèque et Archives Canada de reproduire, publier, archiver, sauvegarder, conserver, transmettre au public par télécommunication ou par l'Internet, prêter, distribuer et vendre des thèses partout dans le monde, à des fins commerciales ou autres, sur support microforme, papier, électronique et/ou autres formats.

L'auteur conserve la propriété du droit d'auteur et des droits moraux qui protège cette thèse. $\mathrm{Ni}$ la thèse ni des extraits substantiels de celle-ci ne doivent être imprimés ou autrement reproduits sans son autorisation.
In compliance with the Canadian

Privacy Act some supporting forms may have been removed from this thesis.

While these forms may be included in the document page count, their removal does not represent any loss of content from the thesis.
Conformément à la loi canadienne sur la protection de la vie privée, quelques formulaires secondaires ont été enlevés de cette thèse.

Bien que ces formulaires aient inclus dans la pagination, il n'y aura aucun contenu manquant.

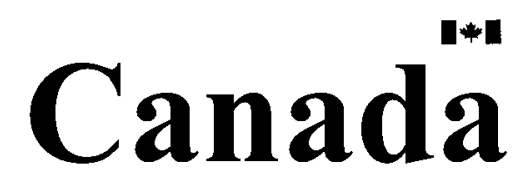




\section{Abstract}

In the absence of any previously published surgical robotic literature in relation to machine vision and autonomous surgical activitiy, this thesis describes the development of an interdisciplinary, conceptual visually autonomous cauterization system (VACS). In general, the thesis identifies a surgical task and niche for which a device may be visually servoed to act in the operating room as a surgical assistant. Specifically, the intent is to provide operating teams with control over hemostatic maintenance through electrocautery while simultaneously conducting other surgical procedures. Cauterization during minimally invasive hand-assisted laparoscopic liver resection (i.e., identified as the most likely procedure for proof-of-concept success) was selected. Design requirements for the system are established and details of the required hardware and software are discussed. The perioperative phases of the handassisted laparoscopic liver resection are outlined, then video frames from one such surgery are analyzed demonstrating the use of the image processing algorithm to identify cauterisable areas. Finally, a discussion on how the system's benefits are realized and conclusions based on the objectives achieved and suggestions for future work (i.e., intended to establish a physical prototype and viable resource for the surgical community) are presented. 


\section{Acknowledgments}

I would like to gratefully acknowledge the enthusiastic supervision of Dr. M. John

D. Hayes. Also, this work would not have been possible without the support of Dr. Tofy Mussivand. It is difficult to overstate my gratitude for both of my supervisors' guidance. Thank you!

Additional thanks to the many people and organizations involved in making this thesis possible, including; the Department of Mechanical and Aerospace Engineering at Carleton University, the University of Ottawa Heart Institute and the Departments of Surgery at both the Ottawa General Hospital and Ottawa Civic Hospital.

Finally, it is a pleasure to thank my parents for their encouragement, patience and support without which this project and many others could not have been a success. My sincere appreciation to Jeffrey Spooner for his untiring efforts in the preparation of this work. I would also like to especially thank Leslie Small, "Thanks mom you're the greatest!". 


\section{List of Symbols \& Acronyms}

$\begin{array}{ll}\text { AESOP } & \text { Automated Endoscopic System for Optimal Positioning } \\ \text { AI } & \text { Artificial Intelligence } \\ \text { CT } & \text { Computed Tomography } \\ \text { FDA } & \text { Food and Drug Administration } \\ \text { INS } & \text { Interactive Network System } \\ \text { IOUS } & \text { Intraoperative Ultrasound } \\ \text { IV } & \text { Intravenous } \\ \text { MIS } & \text { Minimally Invasive Surgery } \\ \text { MST } & \text { Master-Slave Telemanipulator } \\ \text { O.R. } & \text { Operating Room } \\ \text { VACS } & \text { Visually Autonomous Cauterization System }\end{array}$




\section{Contents}

Abstract

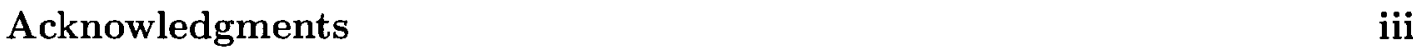

List of Symbols \& Acronyms iv

Contents v v

List of Tables viii

List of Figures viii

Claim of Originality $\quad \mathbf{x}$

1 Introduction 1

1.1 Objectives and Scope . . . . . . . . . . . . . . . . . . 2

1.2 Problem Statement . . . . . . . . . . . . . . . . . . . 3

1.3 Design Solution Requirement \& Benefits . . . . . . . . . . . . . . 4

1.4 VACS Surgical Application . . . . . . . . . . . . . . . 5

1.4 .1 MIS Liver Resection . . . . . . . . . . . . . . . . 6

1.5 Design Concept . . . . . . . . . . . . . . . 11

2 Literature Review $\quad 14$

2.1 Digital Image Processing . . . . . . . . . . . . . . . . . 15

2.1.1 A Robust Vision-based Moving Target Detection and Tracking System . . . . . . . . . . . . . . . . 16

2.1.2 Likelihood-based Object Detection and Object Tracking Using Color Histograms and EM . . . . . . . . . . . . . . 18

2.1.3 Subspace Methods for Robot Vision . . . . . . . . . . . . . 19

2.1.4 CONDENSATION-Conditional Density Propagation for Visual Tracking . . . . . . . . . . . . . . 22 
2.1.5 Video Object Extraction Based on Adaptive Background and Statistical Change Detection . . . . . . . . . . . . . . . 23

2.2 Surgical Technology . . . . . . . . . . . . . . . . . . . 26

2.2 .1 The da Vinci Surgical System . . . . . . . . . . . . 26

2.2.2 Automated Endoscopic System for Optimal Positioning (AESOP) 28

2.2.3 Voice Activated O.R. (Hermes) . . . . . . . . . . . . 30

2.2.4 Smith \& Nephew Endoscopy's Condor Control System . . . . 30

2.2.5 Laparoscopic Graspers with Force Measurement . . . . . . . . 32

3 VACS Design Requirements $\quad 34$

3.1 Size \& Location . . . . . . . . . . . . . . . . . . . . . . . . . . . . . . . . . . . . . 35

3.2 Workspace . . . . . . . . . . . . . . . . . . . . 36

3.3 Portability . . . . . . . . . . . . . . . . . . . . . . . 37

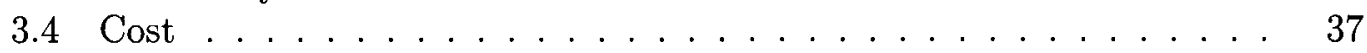

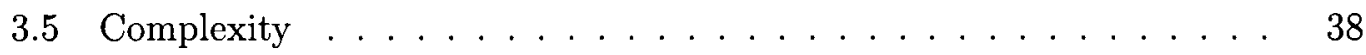

3.6 Sterilization . . . . . . . . . . . . . . . . . . . . 38

3.7 Cautery Probe . . . . . . . . . . . . . . . . . . . . . 39

3.8 Machine Vision . . . . . . . . . . . . . . . . . . . . . . . . . 39

3.9 Voice Command . . . . . . . . . . . . . . . . . . . 40

3.10 Robotic Positioning . . . . . . . . . . . . . . . . . . 40

4 VACS Design Concept 41

4.1 VACS Endoscope . . . . . . . . . . . . . . . . . . 42

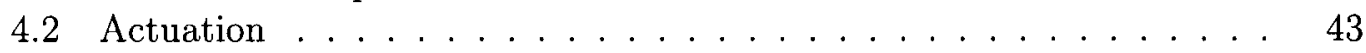

4.3 VACS Cauterization Probe . . . . . . . . . . . . . . . 44

4.3 .1 Electrocautery. . . . . . . . . . . . . . . . . . . . . 45

4.3 .2 Argon Beam Coagulator . . . . . . . . . . . . . . . 48

4.4 Surgical Platform . . . . . . . . . . . . . . . . . . . . . . . . . 48

4.5 Voice Command . . . . . . . . . . . . . . . . . . . . . . . . . . . . . 49

4.6 VACS Feedback System . . . . . . . . . . . . . . . . 50

4.7 VACS Machine Vision . . . . . . . . . . . . . . . . . 51

5 VACS Mechanical Design \& Rapid Prototyping 53

6 VACS Control System $\quad \mathbf{5 7}$

6.1 Surgical Modalities . . . . . . . . . . . . . . . . . . 58

6.1 .1 VACS Modality . . . . . . . . . . . . . . . 59

6.2 Functional Level MST . . . . . . . . . . . . . . . . . . . . . . . . 59

6.3 Artificial Intelligence Decision Tree . . . . . . . . . . . . . 60 
7 Image Processing $\quad 65$

7.1 Problem Description . . . . . . . . . . . . . . . 66

7.1.1 Real-time Medical Imaging . . . . . . . . . . . . . . 66

7.1 .2 Moving Background . . . . . . . . . . . . . . 67

7.1 .3 Moving camera . . . . . . . . . . . . . 67

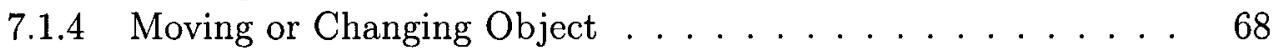

7.1 .5 Clutter . . . . . . . . . . . . . . . . . 68

7.2 Suitable Algorithms . . . . . . . . . . . . . . . . . . 68

7.3 Implemented Algorithms . . . . . . . . . . . . . . . . . . 69

7.3 .1 Colour matching . . . . . . . . . . . . . 70

7.3 .2 Shape matching . . . . . . . . . . . . . . . . 72

7.4 Performance Assessment . . . . . . . . . . . . . . . . 73

8 Discussion \& Conclusions $\quad 75$

9 Recommendations \& Future Work $\quad 79$

Bibliography $\quad 82$

$\begin{array}{ll}\text { A Image Processing Algorithm - Relative } & 90\end{array}$

B Image Processing Algorithm - Absolute 94

$\begin{array}{ll}\text { C Design Drawings } & 99\end{array}$ 


\section{List of Figures}

1.1 Minimally Invasive Surgical Procedures. . . . . . . . . . . . . . . . . 6

1.2 Hand-assisted laparoscopic liver resection. . . . . . . . . . . . . . 9

2.1 Background motion compensation from two consecutive frames. . . . 17

2.2 Results of the background estimation and tracking after 337 frames. . 20

2.3 Tracking with multi-modal state-density. . . . . . . . . . . . . 23

2.4 Example of region segmentation. . . . . . . . . . . . . . . . . 24

2.5 Da Vinci Surgical System Components. . . . . . . . . . . . . . . . . 29

2.6 Voice Controlled O.R. devices. . . . . . . . . . . . . . . . . 31

2.7 Smith \& Nephew Endoscopy's Condor Control System. . . . . . . . . 32

2.8 Laparoscopic Graspers with Force Measurement. . . . . . . . . . . . . 33

3.1 Optic workspace. . . . . . . . . . . . . . . . . 37

4.1 ProE model of VACS endoscope design. . . . . . . . . . . . . . . 42

4.2 VACS endoscope actuator and assembly model. . . . . . . . . . . . 44

4.3 Laparoscopic cautery instrumentation. . . . . . . . . . . . . . . 45

4.4 Liver cautery experimental results for varying cautery probe insertion lengths. . . . . . . . . . . . . . . . . 46

4.5 Valleylab Electro Surgical Unit . . . . . . . . . . . . . . . . . 47

4.6 Potential surgical platforms. . . . . . . . . . . . . . . . . 49

4.7 Speech Controlled Robotic Arm kit. . . . . . . . . . . . . . . 50

4.8 Load cell feedback component and ProE assembly model. . . . . . . . 52

5.1 Distal tip of a flexible bronchoscope. . . . . . . . . . . . . 55

5.2 3D ProE model of the VACS endoscope. . . . . . . . . . 56

6.1 Modalities used in different configurations for performing surgery . . 62

6.2 Modalities used in conjunction with VACS . . . . . . . . . . . . 63

6.3 VACS control system expressed using MST subcategories. . . . . . . . 63

6.4 Artificial intelligence decision tree. . . . . . . . . . . . . . 64

7.1 Video frame captured from hand-assisted laparoscopic liver resection $\quad 67$ viii 
7.2 Relative frame processing sequence. . . . . . . . . . . . . 70

7.3 Absolute frame processing sequence. . . . . . . . . . . . . 72

C.1 VACS endoscope design drawing. . . . . . . . . . . . . . . . . . . 100

C.2 VACS endoscope adaptor design drawing. . . . . . . . . . . . . . 101

C.3 HSI 21000 Series - Size 8 Non-Captive Actuator Linear design drawing. 102

C.4 Honeywell miniature load cell design drawing. . . . . . . . . . . . . . 103

C.5 VACS cautery probe design drawing. . . . . . . . . . . . . 104 


\section{Claim of Originality}

To the best of my knowledge, certain aspects of this thesis are presented herein for the first time. Specifically, these contributions of the Visually Autonomous Cauterization System (VACS) include:

1. the VACS endoscope conceptual design;

2. the VACS actuation and cautery probe design and first prototype constructed using ProE drawings to generate ABS plastic components in a $3 \mathrm{D}$ printer;

3. the VACS machine vision algorithm conceptual design. 


\section{Chapter 1}

\section{Introduction}


Given significant advances in surgical robotics $[1,2,3]$ together with increasing popularity of minimally invasive surgery [4], demand for more sophisticated technologies, capable of enhancing or extending current proficiencies $[5,6]$ is imminent. It is anticipated that the next generation of surgical robotics will be required to provide greater autonomous support [7]. Advances in this area will help reduce surgical durations, thereby reducing patient time under anesthesia, operating room allotment periods, and ultimately, patient stays. One such opportunity rests with the hemostatic maintenance required during laparoscopic and thoroscopic procedures accomplished by detection and cauterization (i.e., a task routinely designated to surgeons).

The proposed development of a visually autonomous cauterization system (VACS) will provide operating teams with simultaneous control over this compulsory task while freeing them to concentrate upon other procedures. VACS will combine existing surgical robotic technology with machine vision advances enabling voice-activated and image-guided precise surgical movements.

\section{$1.1 \quad$ Objectives and Scope}

The objectives of the VACS project are as follows: develop a conceptual design for a semi-autonomous robotic surgical assistant with a current application to cauterization; complete conceptual and detail design of an endoscopic probe; complete endoscopic probe prototype construction; develop elementary image processing feature recognition software and perform feasibility testing; identify existing surgical robotic platforms for which VACS can act as an autonomous extension; establish the feasibility of a visually semi-autonomous surgical assistant capable of performing cautery; design and fabricate a prototype endoscope compatible with current surgical demands, 
and those of the VACS; identify the surgical application for which VACS is both capable and feasible; identify the experimental surgical niche in which the VACS can be tested; deduce, through consultation with medical professionals and medical policy, the acceptability and acceptable limits of autonomy for a semi-autonomous cautery surgical assistant.

This work is the first instance where a visually autonomous surgical robotic assistant has been though of. Given that, there is no template in the literature for a VACS-type design, one must start at the conceptual design stage. The work presented in this thesis is intended to be a cornerstone for subsequent development of the ideas and initial elementary results presented herein. The intention is not to present a working prototype system, rather to establish boundaries, and constraints for future development.

\subsection{Problem Statement}

Cleaning up after or during any project is often the most under-anticipated and time-consuming task. Surgical procedures are no different. A substantial amount of time and medical resources, including personnel and equipment are expended in the maintenance of hemostasis during surgical procedures. There is no denying the necessity of this particular task, however, the relative "value added" contribution is minimal at best compared to other surgical activities. Reducing or eliminating the need for the surgical team to invest valuable time and resources into hemostatic maintenance would prove to be considerably beneficial to both patients and surgical care providers, according to consultation with surgical experts.

As the variety of surgical procedures performed with minimally invasive surgery 
(MIS) technology expands, so do the requisite equipment demands and necessary skill levels. Laparoscopic appendectomies, hernia repairs, colon resections and numerous other laparoscopic and thoroscopic procedures all require careful cauterization control. In most cases, this responsibility either falls upon the surgeons themselves or, if available, their surgical residents.

\subsection{Design Solution Requirement \& Benefits}

The general design solution for a visually autonomous cauterization system (VACS), should provide the operating surgeon with simultaneous control over specific compulsory cauterization tasks while physically enabling he/she to concentrate on other aspects of the procedure at hand, thereby enhancing the surgeon's capabilities. Toward this end, VACS is intended to supplant manual detection and cauterization currently employed for hemostatic maintenance during laparoscopic and thoroscopic procedures.

At the same time, these technological advancements must not undermine or challenge the principles of surgery. Moreover, robotic support will never replace human judgment or experience. Rather, these enhancements will continue to revolutionize and improve patient care, and ultimately, well being.

Benefits to a visually autonomous cauterization system described above include:

- Alleviation of specific surgical cauterization demands;

- Relief of designated cauterization personnel;

- Decrease of surgical duration;

- Expected reduction of patient time under anesthesia; 
- Potentially reduced costs.

\subsection{VACS Surgical Application}

Figure 1.1 illustrates several examples of MIS procedures where VACS could prove to be surgically advantageous given it's ability to cauterize bleeding areas independently from other, more directed, surgical tasks. Figure 1.1(a) shows the mobilization of a right lung (right) from the chest cavity wall (left) during a right pneumonectomy, during which bleeding occurs from tissue growth connecting the two structures. Figure 1.1(b) shows the surgeons hand (left - white) holding the liver during a handassisted liver resection while a surgical colleague cauterizes the liver (centre) with argon beam coagulation. The adjustable banding procedure (seen in Figure 1.1(c)) involves suturing a semi-elastic band around the stomach pouch, thus reducing it's effective volume. In order to assure that the band remains in place, sutures are used to secure the band to the exterior of the stomach. Figure 1.1(c) shows bleeding as a result of the suturing process.

Although all of these MIS procedures represent viable options for VACS development, MIS liver resection was selected as the initial surgical niche. Resection of the liver presents a unique array of suitable surgical demands. Chief among these, given the liver's major detoxification responsibilities involving adjustments to blood chemistry, is that the liver is highly perfused with blood (i.e., thus making the resection of the liver an ideal location for VACS to control bleeding). 


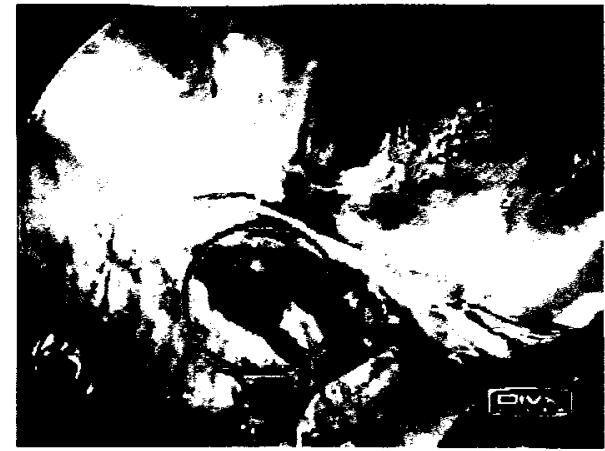

(a) Video assisted thoracic lobectomy.

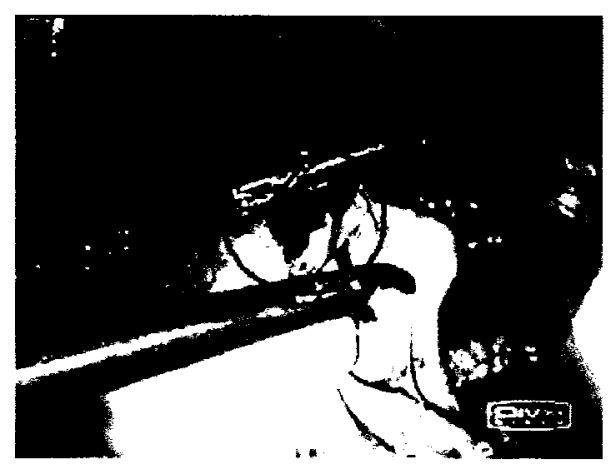

(c) Laparoscopic Adjustable Banding Procedure.

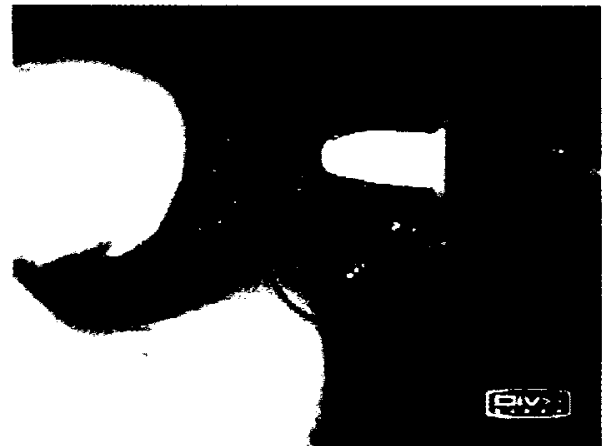

(b) Laparoscopic liver resection.

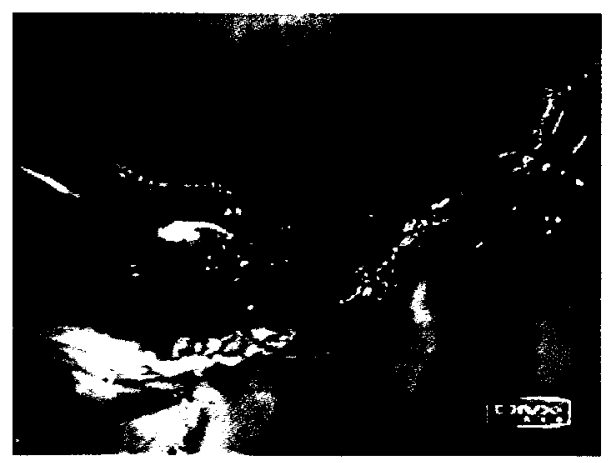

(d) Laparoscopic Supracervical Hysterectomy.

Figure 1.1: Minimally Invasive Surgical Procedures [8]. These images taken from a variety of MIS procedures clearly shows potential locations for cauterization that could be performed concurrently with other surgical tasks.

\subsubsection{MIS Liver Resection}

MIS liver resection is a common laparoscopic surgical procedure typically performed to extract damaged or cancerous portions of the organ without creating an excessively gaping incision. Minimally invasive liver resection involves inserting a small endoscopic camera alongside several long, thin rigid instruments through small incisions in the abdomen. The surgery is performed by manipulating instruments extracorporealy while simultaneously viewing the intracorporial results on a video screen 
displaying the endoscopic video feed.

The following comprises a perioperative overview of a typical MIS liver resection:

\section{Preoperative}

Before a liver resection is performed, all prospective patients must be evaluated to ensure that they qualify as suitable candidates for the procedure. $\mathrm{A} \mathrm{CT}^{1}$ scan is typically performed prior to surgery in order to assess the size and location of the area to be resected as well as the existence of hepatic cysts and/or benign hepatic lesions or malignancies. A CT scan also provides surgical teams with an opportunity to search for extrahepatic (i.e., outside the liver) tumors. If found, the resection may still be performed in order to relieve symptoms caused by the cysts, lesions or tumors. If, however, cancer is found to exist in other inoperable regions of the body, it may be presumed that proceeding with a resection will likely do little to improve the patient's odds of survival. Under these circumstances, decisions to proceed are made on a case by case basis.

Typically, as with most major surgeries, once the patient arrives in the operating room, but prior to the commencement of surgery, the anaesthetist attaches several $\mathrm{IVs}^{2}$. In addition, an epidural anaesthetic tube may also be placed in the spine to deliver medication intra/postoperatively. A catheter may also be inserted into the bladder in order to drain urine, depending upon the expected duration of the procedure [10].

\footnotetext{
${ }^{1} \mathrm{CT}$ (computed tomography), sometimes called CAT scan, (i.e., using specialized x-ray equipment to obtain image data from varying angles around the body) computes and processes the accumulated data using information to generate and display a cross-section of body tissues and organs [9].

${ }^{2} \mathrm{~A}$ method of administering fluids and medications via a needle inserted into a vein.
} 


\section{Intraoperative}

Gamlin et al. provides a more extensive summary of laparoscopic liver resection [11]. The availability of hand-assisted laparoscopic surgery exists as a safe and feasible option for the removal of wedge segments of liver [12]. It is now possible to perform resection on any part of the organ provided that the tumor is present on the surface. Manipulation with the hands allows access to all parts of the liver [13]. Figure 1.2 shows a hand-assisted laparoscopic liver resection. The hand-assisted laparoscopic wedge resection (i.e., as illustrated in Figures 1.2) provides an excellent cauterisable area for the VACS proof-of-concept surgical application.

Laparoscopic intraoperative ultrasound (IOUS) examination of the liver during surgery may also be performed. This procedure provides up-to-date information about the tumor and may detect additional abnormalities that were not initially observed in the original CT studies performed prior to surgery $[11,13]$.

Figure 1.2(a) is a video frame taken from the intraoperative endoscopic feed. The major biological feature in the image is the liver. The surgical tool in the frame is a MIS stapling device. This device, operated by the surgeon, is first used to grasp the tissue along the line of resection. After the tissue is secured in the jaws, a secondary procedure is executed which simultaneously cuts the tissue lengthwise along the jaws and staples both sides of the incision. The rows of staples are used to help minimize bleeding after the jaws are released and blood is able to flow back to the incision (Note: Due to the thickness of the liver, however, the $5 \mathrm{~mm}$ staples are incapable of controlling the bleeding, as seen in Figure 1.2(b). Hence the need for additional cauterization techniques, including; electrocautery, argon beam cautery and VACS.). 


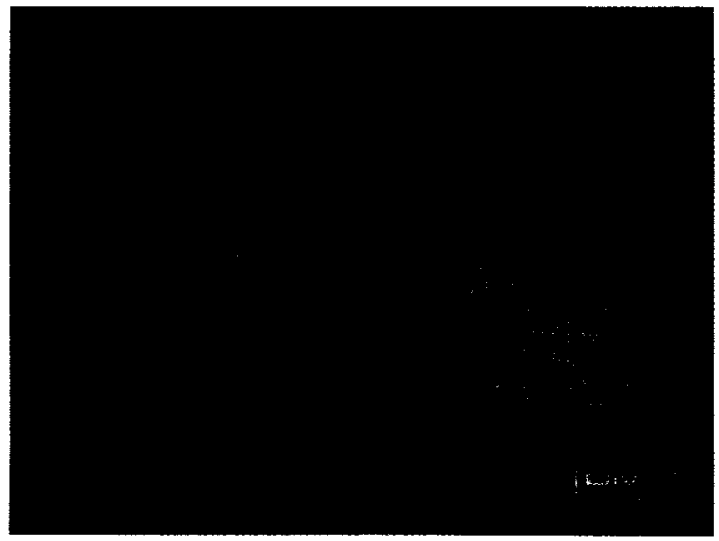

(a) Hand-assisted laparoscopic wedge resection using an articulating stapler.

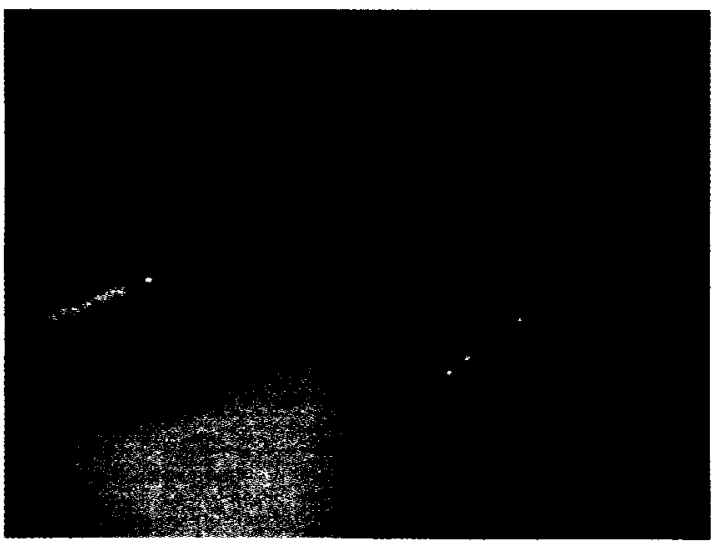

(b) Cauterisable bleeding plane of the liver.

Figure 1.2: Hand-assisted laparoscopic liver resection [8].

\section{Postoperative}

Unlike most internal organs, the liver possesses the ability to regenerate itself [14]. In fact, following a resection and provided that the remaining portion is healthy ${ }^{3}$, the liver may regenerate to it's preoperative size within 2 weeks $[15,16]$. For this reason, it is possible to safely extract up to $75 \%$ of the organ's tissue [17]. The typical hospital stay following a resection is approximately 5 days. Most patients complete a full recovery within 5-6 weeks (i.e., from a 75\% resection). This somewhat. unique regeneration capability offers surgeons a great deal of additional confidence when performing liver resections.

\footnotetext{
${ }^{3} \mathrm{~A}$ cirrhotic liver, for example, cannot revive itself. Therefore, before a resection is performed to excise a malignancy, the non-tumor portion of the liver may be biopsied to determine whether there is associated cirrhosis.
} 


\section{Bloodless Surgery}

One of the major challenges in resecting larger portions of the liver is controlling blood loss. Bloodless surgery represents a major trend in modern medicine and is supported by a large portion of the medical community $[18,19,20]$. VACS can play an important role in minimizing blood loss during procedures. Given the ability to perform autonomous cauterization, the system coagulates blood vessels in order to reduce blood loss and minimize operative time. Both open and MIS liver resection take approximately 3-5 hours and may be performed without the need for a blood transfusion provided that efforts are undertaken to closely monitor and maintain hemostasis [17].

The preceding discussion is underscored by the following quotation from Drs. Rick Selby and Nicolas Jabbour at the USC University Hospital Center for Liver Disease.

"The practice of bloodless medicine involves the delivery of medical and surgical care without the transfusion of blood products. This technique has long been applied to Jehovah's Witness patients, but is now being used more extensively with most patients due to the lessened risk of infection and immunologic complications.

Liver transplant surgeons Selby and Jabbour at the USC University Hospital Center for Liver Disease use a multidisciplinary approach to eliminate the need for the transfusion of blood and blood products. Their two-fold strategy, honed over 10 years, involves building up the body's own reserve of blood components prior to surgery and then conducting all operative procedures in a manner that minimizes blood loss. Of critical importance is the identification of distinct anatomic planes and the rapid control of 
even minor hemorrhage using surgical ligation and local agents that promote coagulation in the operative field. Liver transplant surgery is a long and complicated procedure in which all efforts must be made to reduce stresses on the patient. Keeping blood loss to a minimum, therefore, is of vital importance.

Once the surgeons recognized the patient benefits involved in bloodless surgery during transplantation, they began applying the same techniques to all abdominal surgical procedures they performed. Regardless of whether patients were undergoing surgery for simple hernias or complex liver or pancreatic surgeries, the bloodless surgical technique was used and the beneficial results of such were immediately obvious. Perhaps most noteable was the fact that the frequency of post-surgical infections was lessened, as was patient recuperation time. " [18]

\subsection{Design Concept}

The VACS design concept consists of several elements, specifically:

1. Endoscope;

2. Video Adaptor;

3. Actuation;

4. Cautery Probe;

5. Machine Vision Algorithm and Control System; 


\section{Surgical Manipulation Platform.}

The VACS endoscope design concept represents a simple, but innovative adaptation of existing laparoscopic equipment. While based on a standard rigid laparoscope, it introduces a designated instrument channel which serves as a conduit for a cautery probe (e.g., bipolar electrocautery or argon beam cautery) running parallel to the longitudinal axis of vision. The cautery probe is extended and retracted through a computer controlled, non-captive linear actuator mounted to the rear of the VACS endoscope.

The machine vision software algorithm provides the foundation of artificial intelligence for the VACS, based on the processing of individual video frames from the VACS endoscope. This algorithm was applied to the laparoscopic video feed of a hand-assisted laparoscopic liver resection [8]. Such image processing enables the identification of bleeding structures that can then be cauterized by the VACS at the surgeon's discretion.

The VACS design concept requires a robotic system capable of positioning the VACS endoscope based on feedback from the VACS machine vision algorithm, potentially initiated through voice commands from the surgeon. Both the da Vinci Surgical System [1] and the AESOP Endoscope Positioner [21] satisfy this requirement and have been approved for use by the United States Food and Drug Administration [22].

In this visually autonomous system the operative tasks involved are performed under a surgeon's supervision, yet without the need for direct physical contact, hence eliminating tremors, strain and fatigue. The two-fold benefit involves not only enhancing a surgeons individual capabilities but enabling the entire team to focus on more challenging operative tasks. 
Based on the above description, the conceptual design proposes the following order of operations for the visually autonomous cauterization system:

Identification of potential target for cauterization through image processing of the surgical video feed;

Discrimination between potential targets by the surgeon to safely identify appropriate areas for VACS to execute the cauterization task;

Communication between the surgeon and VACS through voice commands to initiate and control the physical tasks of cauterization; and

Cauterization of the desired surgical area. 


\section{Chapter 2}

\section{Literature Review}


In this chapter the literature reviews of both the digital image processing and the surgical robotic technology are presented.

\subsection{Digital Image Processing}

Object detection and tracking remains an open research problem. The digital image processing requirements in this thesis concerns real-time moving objects, moving backgrounds and moving observer scenarios. The challenge here depends on how the object to be detected and tracked is defined. Most difficulties stem from the image variability, particularly for video because moving objects are generally involved. As an object moves through the field of view of a camera, the features of the object may change dramatically. This scenario will require a robust, real-time, generalized approach to identify and track the objects of interest.

It is well known that detection and tracking of abstract targets is a very complex problem and demands sophisticated solutions using pattern recognition and motion estimation methods [23]. Although large amounts of research have been done in the the area of target detection and tracking, a generalized approach that satisfies all possible scenarios and conditions has not been defined. However, there appear to be methods that are capable of satisfying individual aspects of the digital image processing requirements (e.g., object detection, object tracking, background elimination, etc.). Therefore, combining multiple methods should allow the difficulties to be overcome. 


\subsubsection{A Robust Vision-based Moving Target Detection and Tracking System}

Behrad et al. present a new algorithm for real-time detection and tracking of moving targets in terrestrial scenes using a mobile camera [24]. In this case the algorithm consists of two parts; one developed for detection and another developed for tracking.

The algorithm developed for target detection used the LMedS (Least median squared) method and affine transformations for robust background motion estimation. In order to determine the background motion, at least three points must be selected from the background. To do so, a modified Moravec ${ }^{1}$ operator was used to find $\mathrm{N}$ distinguishable feature points. This ensures a precise match between frames. Assuming that the objects of interest in the frame are less than $50 \%$ of the whole image, most of the $\mathrm{N}$ points will belong to the stationary background. Selecting $\mathrm{M}$ sets of three feature points from the $\mathrm{N}$ points and computing their corresponding affine transformation gives $\mathrm{M}$ affine transformations. The LMedS method is used to select the optimal transformation, predicting the background motion from the transformation. The 2-D affine transformation is described as follows:

$$
\left[\begin{array}{l}
X_{i} \\
Y_{i}
\end{array}\right]=\left[\begin{array}{ll}
a_{1} & a_{2} \\
a_{3} & a_{4}
\end{array}\right]\left[\begin{array}{l}
x_{i} \\
y_{i}
\end{array}\right]+\left[\begin{array}{l}
a_{5} \\
a_{6}
\end{array}\right]
$$

Following the background motion estimation, it is possible to identify real moving objects. This is accomplished by canceling the background motion. Therefore, the differences between the current frame and the transformed previous frame reveals the

\footnotetext{
${ }^{1}$ The Moravec operator selects pixels with the maximum directional gradient in the min-max sense
} 
moving objects, see Figure $2.1^{2}$ where the first two images presented are consecutive frames taken from a video of a car driving away from the camera along a treed road with a building visible in the background. In the third image, a background elimination technique was used to identify pixels between the two frames as per the above criteria. As such, those pixels were deemed to be background, and are represented by the black area of the third image. The white pixels of the third image are pixels representing a location were pixel intensity between the two video frames are not classified as background, thus indicating a region of motion. The split and merge $^{3}$ technique is used to identify target bounding boxes. In this case, the objects of interest were moving vehicles, which can be fairly well characterized by aspect ratio and horizontal and vertical lines.
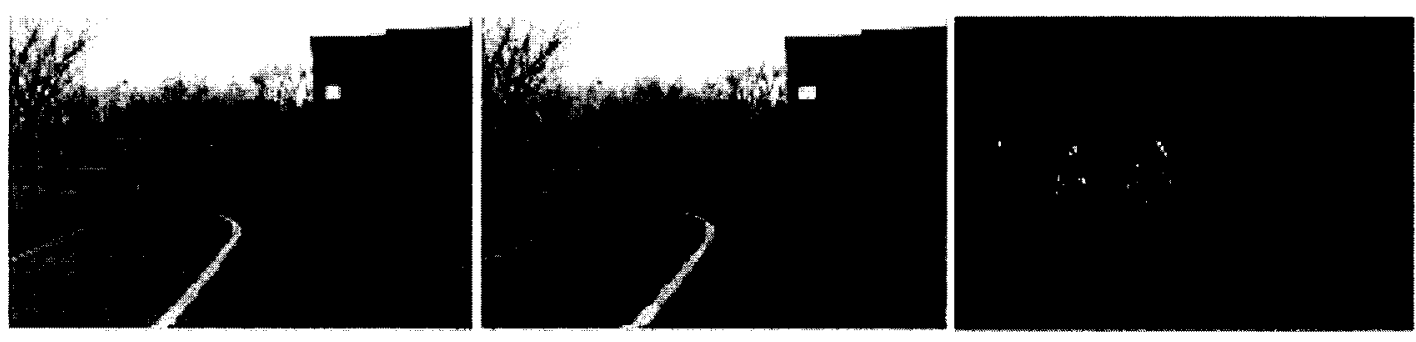

Figure 2.1: Background motion compensation from two consecutive frames [24].

Once target detection and verification has been completed, the algorithm switches to the tracking mode. Once again, a modified Moravec operator is applied to the target, identifying feature points that can be used to track the target. The feature points, in conjunction with disparity vectors ${ }^{4}$ compared between frames, help to refine

\footnotetext{
${ }^{2}$ The calculated affine parameters are: $\mathrm{a} 1=0.9973, \mathrm{a} 2=-0.004, \mathrm{a} 3=0.008, \mathrm{a} 4=1.0022, \mathrm{a} 5=1.23$, $\mathrm{a} 6=-2.51[24]$.

${ }^{3}$ Split and Merge Segmentation: recursively splitting the image into smaller and smaller regions until all individual regions are coherent, then recursively merging these to produce larger coherent regions [25].

${ }^{4}$ Disparity or difference vectors are computed for the matched pairs of points between frames.
} 
the points that define the new position of the target in the scene. In the event that the target is lost or missed, the algorithm switches back to the detection mode. Although the detection mode could also be used to track targets, the tracking algorithm does so with low computational cost.

In addition, once in tracking mode, the object can become larger than $50 \%$ of the image and still be tracked. This is because there is no need to estimate background motion after target feature points have been identified.

This multi-mode algorithm is capable of detecting and tracking targets in realtime. When run on a Pentium III 500Mhz using a Visual $\mathrm{C}++$ program a frame rate of between 4 and 15 frames/second was achieved for $352 \times 288$ pixel video frames.

\subsubsection{Likelihood-based Object Detection and Object Track- ing Using Color Histograms and EM}

Withagen et al. discuss the integration of Expectation Maximization (EM) background modelling and template matching using colour histograms as templates to improve tracking for surveillance purposes [26].

Surveillance applications typically consist of moving object detection and tracking. Tracking non-rigid bodies, such as humans, poses a difficult problem because the algorithms must allow for shape deformation. This task is accomplished by applying a likelihood-based framework to help decide which pixels belong to the background or an object.

A technique called Expectation Maximization is used to model the background. This is an adaptive technique that allows the model to change over time. For each 
pixel, the likelihood that it belongs to the background is calculated. Once the likelihood calculations are complete, the results are thresholded to distinguish foreground from background. Objects are modelled as color histograms since histogram-based object recognition has the advantage of being invariant under small deformations.

In addition, object models are constantly updated to maintain a proper template. In order to avoid corruption of the template by occlusion or a poor estimate of the object, the template is only updated with a fraction of the actual histogram.

Object tracking is done in several stages. First, histogram matching is used to identify the most likely location of the object. Second, binary erosion ${ }^{5}$ is used to identify the object-core and a comparison between the two frames and the predicted object-core is completed. This is followed by updates to the object's location based on absolute differences between the two frames. Binary dilation ${ }^{6}$ is then used to determine which pixels belong to the object. See Figure 2.2 for illustration, where A and B are two images from earlier in the sequence (frames 235 and 285). C shows the current input image (frame 337) and D the related background likelihood. E shows three tracked blobs (whitc) with their boundary (colored), a bounding rectangle, and the path the objects have made in the past (squares and circles). F shows the input frame with the same information as in E overlayed.

\subsubsection{Subspace Methods for Robot Vision}

Nayar et al. discuss a non-traditional approach to visual recognition in [28]. Visual recognition is typically based on shape matching. This approach matches appearance

\footnotetext{
${ }^{5}$ Binary erosion: surrounding pixels that bridge objects can be eroded away by systematically removing edge pixels [27].

${ }^{6}$ Binary dilation: pixels may be added systematically to fill holes and close spaces between objects [27].
} 

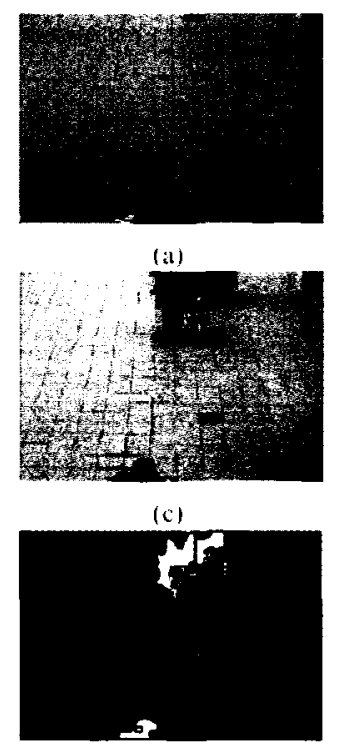

(c)
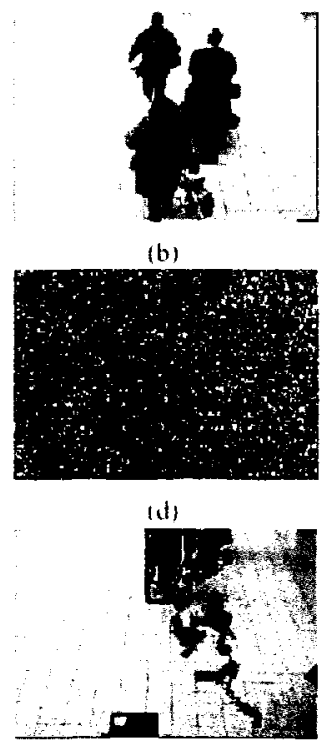

(i)

Figure 2.2: Results of the background estimation and tracking after 337 frames [26].

parameters, including: brightness, reflectance, illumination, and shape. In order to implement shape recognition, a vision programmer must develop models of objects and manually input this information into the system. This becomes impractical for large scale operations, and demonstrates the need for future vision systems to have the capability of acquiring object models themselves. The appearance model being proposed in [28] is capable of generating a model through an automatic learning phase. Drawing on subspace methods, it is possible to acquire and recognize appearance models efficiently in a lower-dimensional subspace called the eigenspace.

The underlying techniques of the appearance based approach can be applied to a number of vision problems, including: visual positioning or servoing of robotic manipulator and real-time tracking. Almost any application relying on image recognition has the potential to be conducted using the appearance based approach. 
Implementing this approach involves two phases. Prior to the image recognition phase, a learning phase must be completed to map the visual workspace to the eigenspace. This is accomplished by generating a large number of object images by incrementally displacing the camera. In this particular case, the camera is mounted to a robot end-effector. These images, and the appearance characteristics imbedded in them, are compacted into the eigenspace. The variation in the object images created by displacing the robot are represented as a parameterized manifold ${ }^{7}$ in the eigenspace.

The second phase of the process is image recognition. This is where the efficiency of the appearance based approach becomes evident. Traditional methods require a brute force approach to compare the input image with all the images corresponding to the workspace samples. This is highly impractical from a computational perspective due to the large number of images. In addition, the input image may not match exactly with those from the sampling. Rather it may lie in between the workspace samples. The eigenspace associated with the appearance based approach can be used efficiently for image matching. The input image is projected into the eigenspace, such that, the closest point on the workspace manifold represents the location of the image.

This technique can also be used to implement a visual positioning algorithm. After image projection into the eigenspace, the deviation on the manifold shows the displacement of the end-effector from the desired position. The appearance based approach avoids the requirement of precise feature detection and matching for visual positioning. It should also be noted that during real-time tracking, assuming the manipulator is near the desired location, fewer learning samples are needed.

\footnotetext{
${ }^{7} \mathrm{~A}$ manifold is a set of points such as those on a closed surface. In this case, a continuous representation of the visual workspace's parameters.
} 


\subsubsection{CONDENSATION-Conditional Density Propagation for Visual Tracking}

Isard et al. present the CONDENSATION algorithm as a means of tracking curves in visual clutter [29]. The CONDENSATION algorithm is based on a stochastic framework of factored sampling that was first developed for static images. The sampling algorithm will be used to track curves, learned from dynamic models, in substantial clutter at video frame-rate.

The main challenge associated with this task is the background clutter. Elements of the background clutter can mimic, occlude, camouflage, and in worst cases, appear similar to the foreground object (i.e., a person moving past a crowd).

A probabilistic model of the object space is defined by low-dimensional B-spline curves [30] (i.e., being careful to avoid any ambiguity but general enough to provide a sufficiently robust definition of the foreground object). Once the object has been located using the probabilistic model, the algorithm generates an observational/state density. The CONDENSATION algorithm, as defined above, is based on factored sampling. This factored sampling is conducted on the state density curve. In the case of real-time video, it is applied iteratively to successive images in the sequence and develops a new state for each image. This new state is created directly only on the immediately preceding state and is unrelated to any other earlier history. Figure 2.3 illustrates an example, where an approximate depiction of the state-density is shown, computed by smoothing the distribution of point masses in the CONDENSATION algorithm. The density is, of course, multi-dimensional; its projection onto the horizontal translation axis is shown here. The initial distribution is roughly Gaussian but this rapidly evolves to acquire peaks corresponding to each of the three people in the 
scene. The right-most peak drifts leftwards, following the moving person, coalescing with and separating from the other two peaks as it moves. Having specified a tracker for one person we effectively have, for free, a multi-person tracker, owing to the innate ability of the CONDENSATION algorithm to maintain multiple hypotheses.

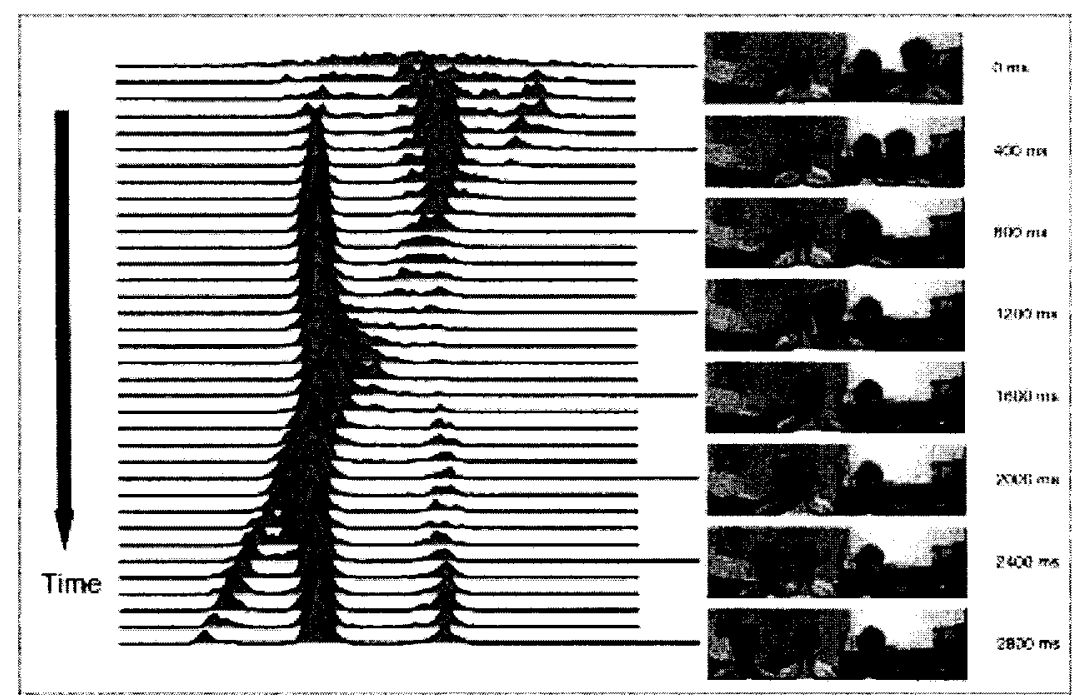

Figure 2.3: Tracking with multi-modal state-density. [29].

\subsubsection{Video Object Extraction Based on Adaptive Background and Statistical Change Detection}

Cavallaro et al. describe a hybrid algorithm for tracking video objects [31]. This hybrid approach uses both object and region information, including low level descriptors, to accomplish the track management task. This video object extraction is classically broken down into two parts: video object segmentation and video object tracking. 
The algorithm works as follows. The video input is received by the object segmentation module. This module produces object partition information based on a change detection method - the design of which is immune to sensor noise [32]. The resulting object partition is a series of masks identifying objects from the background and identifying areas of the image containing moving objects.

Following object partitioning, the region segmentation step only considers areas identified in the object partition. Regions are then identified as areas having homogenous characteristics, see Figure 2.4. These homogeneous characteristics are based on a multi-feature clustering approach focusing on spatial and temporal features.

After the regions are identified, the algorithm generates region descriptors which are used to summarize the value of the features detected in the corresponding region. The tracking management module operates on these region descriptors, using motion compensation, data association and a labelling stage. This provides a more computationally efficient tracking method than tracking the entire region.

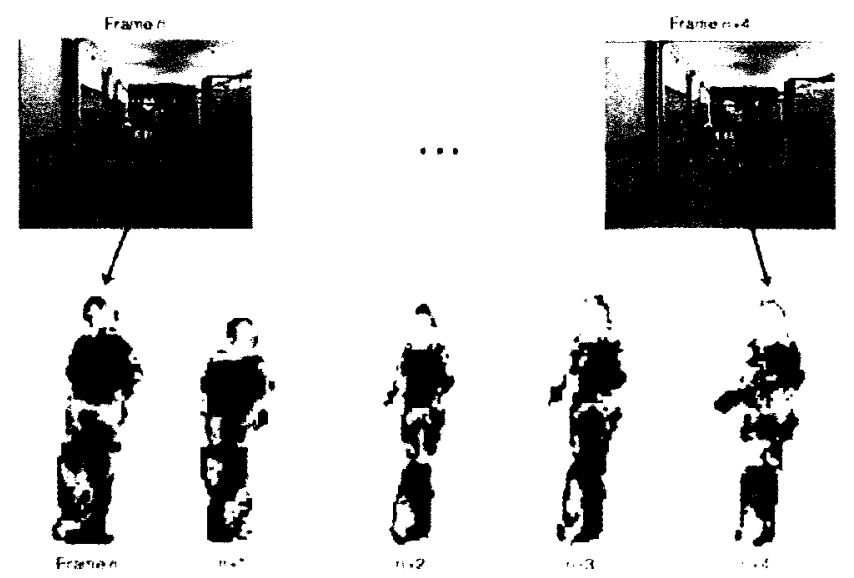

Figure 2.4: Example of region segmentation. Homogeneous regions are computed in each object based on motion, color and texture information [31]. 


\section{Critical Comparison}

All algorithms discussed show excellent results for their intended applications. However, the application with this thesis involves a moving camera and moving scene. In this case, the object for detection will be areas requiring cauterization during laparoscopic surgery. These make for a very unique application, as the bleeding will appear on the moving scene or background. This specialized situation makes some of the approaches discussed more suitable than others.

- The algorithm discussed in [24] is not appropriate because the background subtraction would eliminate the entire scene in our application. Also, the target tracking algorithm would have difficulty identifying features to track.

- [26] has promise in both the Expectation Maximization modelling and the color histogram approach.

- The subspace approach discussed in [28] is an interesting twist on machine vision, however, the need for a distinctive learning phase for every situation is not suitable in this case.

- The CONDENSATION algorithm could be useful as there is no background modelling, and even in substantially cluttered environments, the algorithm can pick out shapes very efficiently.

- Due to the background estimation used to identify objects and regions, the concepts proposed in [31] are not suitable for the VACS intended purpose. 
As was stated in the introduction, some combination of various techniques may produce the most generalized robust solution to overcome the difficulties of our application. Perhaps a combination of color histogram matching for detection, and CONDENSATION for tracking, could be used. The properties of both these algorithms show promise in both of the proposed applications. Color histogram detection would be appropriate since histogram-based object recognition has the advantage of being invariant to small deformations (the object models are constantly updated to maintain a proper template), while CONDESATION's ability to track rapidly and/or randomly moving objects would also prove useful.

\subsection{Surgical Technology}

\subsubsection{The da Vinci Surgical System}

The da Vinci Surgical System (see Figure 2.5) consists of a uniquely designed surgeon's console, a patient-side cart with four interactive robotic arms, the high-resolution InSite Vision System and proprietary EndoWrist Instruments.

The surgeon using the da Vinci Surgical System operates while seated at a console viewing a stereoscopic image of the surgical field. The surgeon's fingers manipulate the master controls of the $\mathrm{MST}^{8}$ system below the display. The surgeon's hands and wrists are naturally positioned relative to his or her eyes. The da Vinci Surgical System interprets the surgeon's hand movements and translates them into precise surgical movements of the EndoWrist Instruments.

The Patient-side Cart can be configured with either three or four robotic arms,

\footnotetext{
${ }^{8} \mathrm{MST}$ - Master Slave Telemanipulator.
} 
two or three instrument arms and one endoscope arm that execute the surgeon's commands. Laparoscopic arms pivot at the $1-2 \mathrm{~cm}$ operating ports, thus eliminating the use of the patient's body wall for leverage and reducing tissue damage. Supporting surgical team members assist in installing the proper instruments, preparing the 1-2 $\mathrm{cm}$ port in the patient and supervising the laparoscopic arms and tools being utilized. Once the da Vinci Patient Side Cart is situated, the patient's position or orientation cannot be readjusted as during traditional surgical procedures.

The EndoWrist instruments are designed with seven degrees of freedom that reproduce the motions of the human hand and wrist. Quick-release levers speed instrument changes during surgical procedures.

The InSite Vision System, with high-resolution 3-D endoscope and image processing equipment, provides the stereoscopic images of the operative field. Operating images are enhanced, refined and optimized using image synchronizers, high-intensity illuminators and camera control units.

Underscoring the above discussion is the following quote from an Article in "Engadget" [3] about Carlo Pappone head of Arrhythmia and Cardiac Electrophysiology at Milan's San Raffaele University.

"For the first time, a robot surgeon in Italy has carried out a long-distance heart operation by itself.

'This operation has enabled us to cross a new frontier,' said Carlo Pappone, who initiated and monitored the surgery on a PC in Boston, ANSA reported. Pappone is head of Arrhythmia and Cardiac Electrophysiology at Milan's San Raffaele University. 
The 50-minute surgery, which took place in a Milan hospital, was carried out on a 34-year-old patient suffering from atrial fibrillation. Dozens of heart specialists attending an international congress on arrhythmia in Boston also watched.

Pappone has used the robot surgeon in at least 40 operations.

'It has learned to do the job thanks to experience gathered from operations on 10,000 patients,' Pappone said, pointing out that the robot carries the expertise of several human surgeons used to boost its software."

\subsubsection{Automated Endoscopic System for Optimal Position- ing (AESOP)}

The AESOP Robotic System released by Computer Motion ${ }^{9}$ in 1994, AESOP was the first robot to be cleared by the FDA for assisting surgery in the operating room [21]. AESOP is simply one mechanical arm, used by the physician to position the endoscope with foot pedals or voice-activated software. This enables the surgeon to position the camera, leaving his or her hands free to continue operating on the patient. Before availability of AESOP, surgical assistants manually held endoscopic cameras, with fatigue and hand tremors causing frequent problems.

The following quote briefly describes the evolution and significance of the AESOP technology [21].

"In 1994, the AESOP 1000 system became the world's first surgical robot

\footnotetext{
${ }^{9}$ Intuitive Surgical and Computer Motion merged on March 7, 2003 into one company that combines their strengths in operative surgical robotics, telesurgery, and operating room integration, to better serve hospitals, doctors and patients [33].
} 


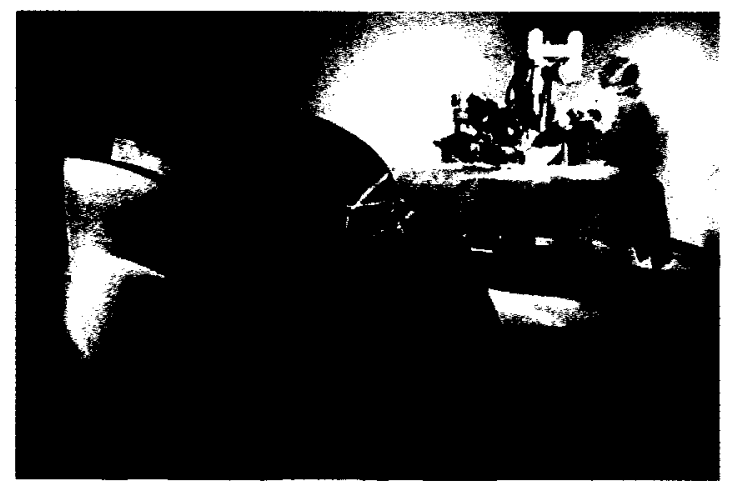

(a) da Vinci Surgical System.

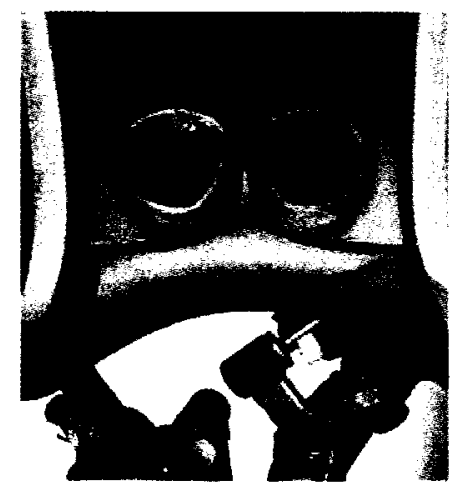

(b) da Vinci Surgical Console.

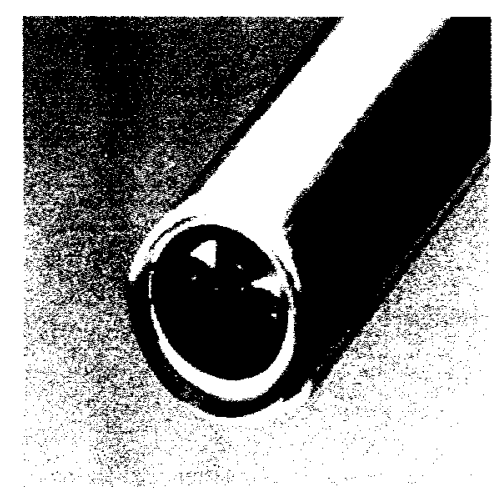

(c) 3D Endoscope.

(c) 3D Endoscope.

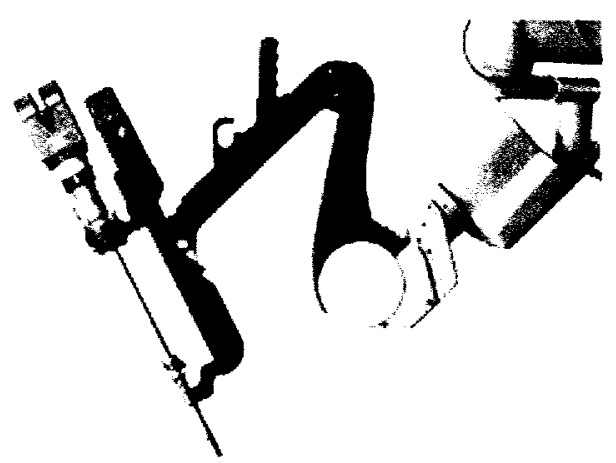

(e) da Vinci Surgical Arm.

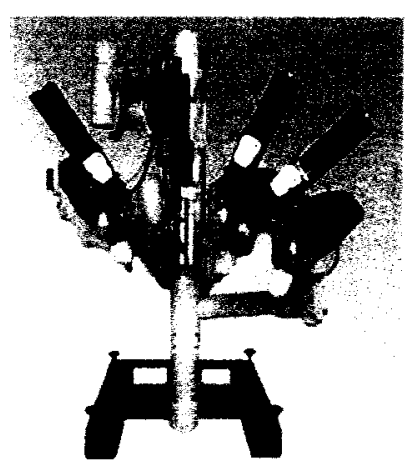

(d) Patient-side Cart.

Figure 2.5: Da Vinci Surgical System Components [1]. 
certified by the FDA in the US. Computer Motion followed with AESOP 2000 in 1996, with the enhancement of voice control, and in 1998, the AESOP 3000, which has 7 degrees of freedom. The redundancy of the AESOP 3000 provided more flexibility in how surgical doctors and nurses can position the endoscope. By 1999, over 80,000 surgical procedures had been performed using AESOP technology."

\subsubsection{Voice Activated O.R. (Hermes)}

Computer Motion's strategy for technology development for the operating room extends beyond robots [34]. They have also developed Hermes, which is a platform for centralizing control of devices inside, as well as resources outside the operating room.

In Computer Motion's vision, all the equipment within the operating room including Computer Motion's robots, other surgical equipment, lights, tables, etc., will be connected to a network through Hermes and can all be controlled using voice commands or a handheld pendant. In addition, surgeons will be able to summon resources such as patient records, test data, etc. outside the operating room.

\subsubsection{Smith \& Nephew Endoscopy's Condor Control System}

Smith \& Nephew's, a medical/surgical device and equipment manufacturer are keenly interested in improving efficiency and patient safety through integrated advanced robotic and voice operated devices in future operating rooms. This is illustrated by the following quote taken from the Smith \& Nephew's Endoscopy news announcement posted online.

"Smith \& Nephew's Endoscopy division announced March 13th, 2006 the 


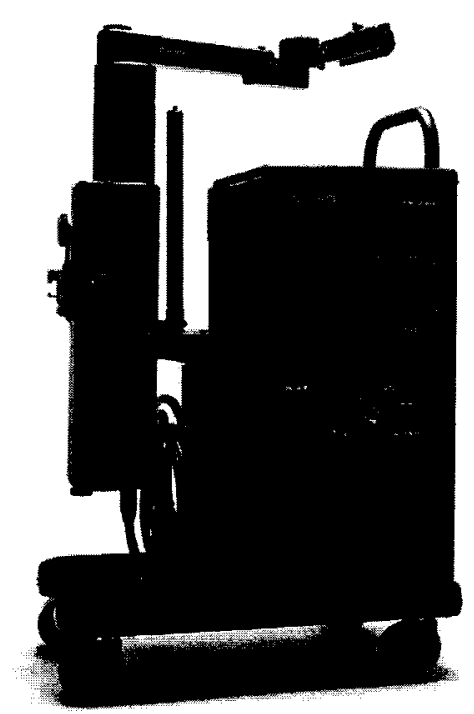

(a) AESOP Cart [21].

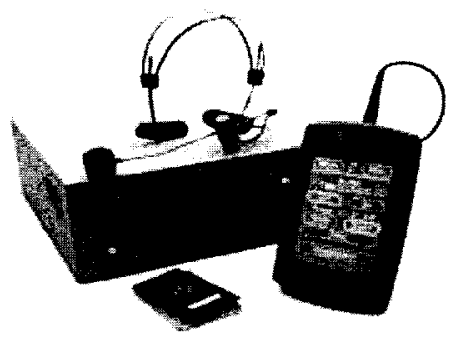

(b) Hermes Sytem [35].

Figure 2.6: Voice Controlled O.R. devices. These devices offer FDA approved, voiceactivated control over a variety of surgical and operating room tasks.

release of the CONDOR Control System for operating rooms, a surgical command center that puts medical staff in control of the devices, patient information and even the lighting and temperature of the operating room. The control system is designed as the hub of Smith \& Nephew's Digital Operating Rooms which are customized surgical solutions designed to improve patient care and operating room efficiency.

"Our new CONDOR Control System enables surgeons and nurses to save time managing surgical devices and devote more time to the job they were trained to do - attend to the needs of patients" said Sal Chiovari, vice president, Digital OR.

CONDOR technology, incorporated into Digital ORs, makes it possible 
for a medical team to send commands to medical devices, digital cameras, image management systems and other components using voice commands and a wireless touch panel. It also enables real-time streaming audio and video of the procedure over the internet to classrooms, offices and consulting surgeons in other locations, around the world.

The system is modular. It can be configured to work with a hospital's existing information systems, and it can be upgraded as new features and new technology are released." [36]

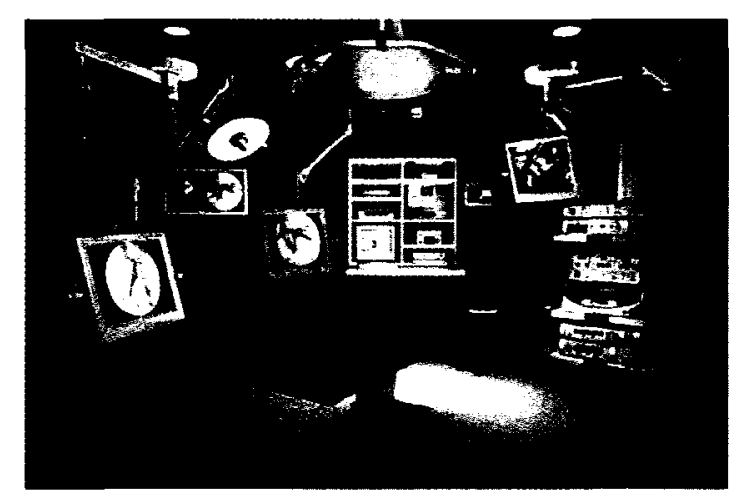

Figure 2.7: The Smith \& Nephew Endoscopy's Condor Control System [36] is envisioned to be highly upgradeable, updateable and to put important patient and surgical information at the finger tips of the surgical team.

\subsubsection{Laparoscopic Graspers with Force Measurement}

Advancements in robotics have led to significant improvements in robot-assisted minimally invasive surgery instruments $[37,38]$. The use of these robotic systems has improved surgeon dexterity, reduced surgeon fatigue and made remote surgical procedures possible. However, commercially available robotic surgical systems do not 
provide any haptic feedback to the surgeon. Just as palpation in open procedures helps the surgeon diagnose the tissue as normal or abnormal, it is necessary to provide force feedback to the surgcon in robot-assisted minimally invasive procedures. Therefore, a need exists to incorporate force feedback in laparoscopic tools for robotassisted surgery. The design of laparoscopic graspers with tri and uni-directional force measurement capability respectively, are described in $[37,38]$ and shown in Figures 2.8. The [37] laparoscopic tool can measure grasping forces and lateral and longitudinal forces, such as those encountered in the probing of tissue. Initial testing of the prototypes has demonstrated the ability to accurately characterize artificial tissue samples of varying stiffness.

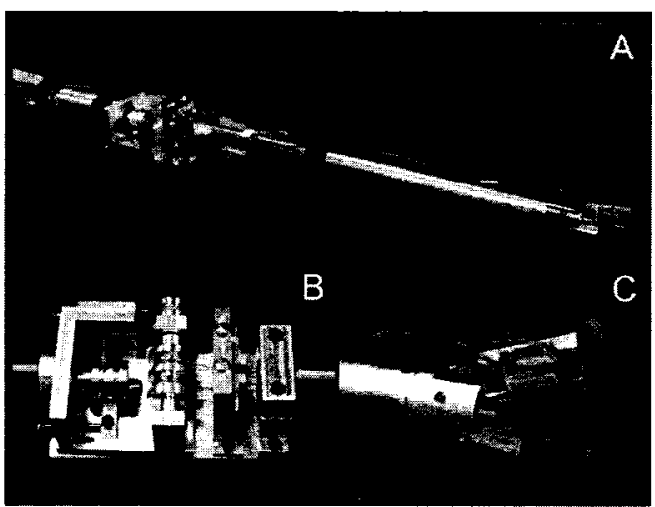

(a) Laparoscopic grasper with 3-D force measurement capability[37].

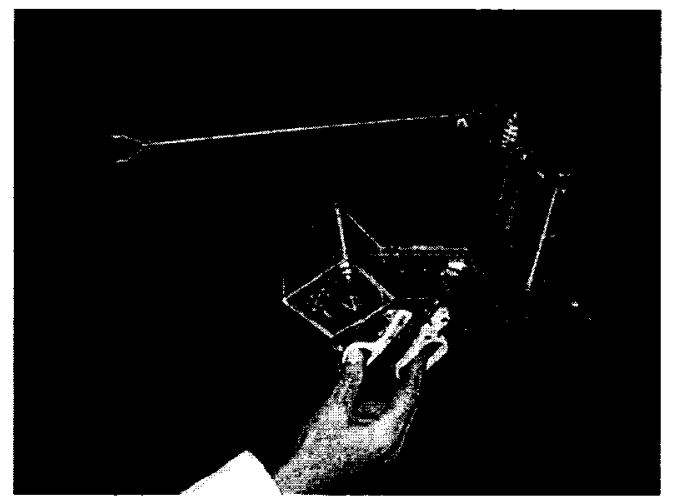

(b) Force controlled and teleoperated Eedoscopic grasper for minimally invasive surgery [38].

Figure 2.8: Laparoscopic gaspers with force measurement. 


\section{Chapter 3}

\section{Visually Autonomous}

\section{Cauterization System (VACS)}

\section{Design Requirements}


A visually autonomous cauterization system will allow operating teams to command a mechanized hemostasis system while simultaneously addressing other essential procedures, such as knot tieing, dissection and/or surgical exploration. VACS will build upon existing surgical robotic technology with machine vision advances, thus enabling voice-operated and image-guided precise surgical movements.

In this chapter the requirements for the VACS conceptual design are detailed.

\subsection{Size \& Location}

Space in an operating room is limited. Often, there can be as many as four surgeons next to the operating table, as well as one or more anesthesiologists, a surgical nurse and a circulating nurse. Oversized medical equipment that further restricts available patient access invariably adds to the confusion, disorganization and stress in the operating room.

Despite the popular acceptance of 'value-to-size' ratio (i.e., that the more useful the equipment, the larger and more cumbersome it can be), smaller is ordinarily preferred. As an example, the footprint of the Patient Side Cart for the da Vinci Surgical System occupies the entire bedside or approximately 6'x3'. And while da Vinci is a MST system designed to be remotely controlled, operating surgeons and nurses are still required to prepare surgical areas and to exchange or insert instruments. Not surprisingly, therefore, a lack of sufficient elbowroom is one of the major drawbacks frequently reported by the $d a$ Vinci operating teams.

The AESOP Endoscopic Positioner is another advanced piece of surgical robotic equipment that offers a more limited range of surgical capabilities than does the da Vinic Surgical System. The AESOP Endoscopic Positioner, in essence, simply 
maneuvers the endoscope during MIS. Accordingly, therefore, and in keeping with the aforementioned value-to-size ratio requirement, the AESOP bedside footprint occupies an area less than half that of $d a$ Vinci's (i.e., approximately 3 'x2').

The VACS System, therefore, while advanced in its abilities - though limited to performing a single surgical task - ought not to occupy more bedside space than the AESOP Endoscopic Positioner. In the interests of valuable space savings, however, it is worth noting that both da Vinci and AESOP posses the voice command, endoscopic vision and dexterous maneuverability necessary for a visually autonomous cauterization system. Consequently, the prospective incorporation of a VACS by either the $d a$ Vinci or AESOP surgical robots would increase their respective operating capabilities along with their relative value-to-size ratios.

\subsection{Workspace}

Munoz et al. [39] discusses the development of a robotic assistant for manipulating the laparoscope during laparoscopic surgery. The camera workspace is defined outside of the abdominal cavity when the endoscope is inserted through the trocar. Figure 3.1 shows the outside limits of the camera positioning, defined by a minimal insertion length of the endoscope through the trocar with the maximal deflection angle of $75^{\circ}$. Therefore, the cartesian workspace of the camera is defined as an inverted cone with a base radius of (a). The camera optic length of $360 \mathrm{~mm}$ is measured from the distal end (d) to camera holder grasping point $(\mathrm{g})$. 


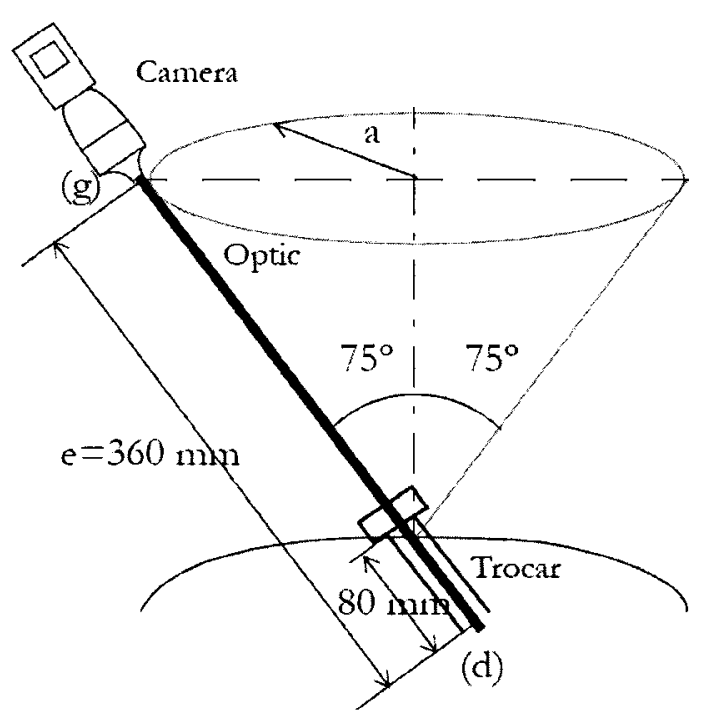

Figure 3.1: Optic workspace discussed in [39] as an inverted cone exterior to the body.

\subsection{Portability}

Modern hospitals that are designed to accommodate surgical care are typically laid out in a similar fashion. Operating rooms are grouped together in an area surrounding the medical equipment and instrument storage areas thus facilitating both the availability and efficient exchange of medical devices and personnel. In addition to critical bedside leeway, therefore, compact equipment is also preferred for its ease of portability. In light of such, portability is given high priority in the VACS design.

\subsection{Cost}

Medical device technology is typically expensive. Nevertheless, development costs for the VACS should be on a par with other costly medical devices, thus allowing the end unit VACS price to be sufficiently enticing so as to not dissuade hospitals 
from investing in the system. Moreover, a modular design is strongly recommended allowing hospitals to acquire individual components on an available funds basis (i.e., rather than being forced to purchase the entire system outright).

\subsection{Complexity}

Increasingly complex devices have a tendency to generate increasingly complex technical and human induced difficulties. As such, the final VACS should avoid, or at least minimize, modifications to standard operating room technologies and procedures in order to reduce confusion and to ease integration. Toward this end, surgeons should be able to conveniently control the movements and execution of most tasks through the use of voice recognition software (e.g., AESOP [21]).

\subsection{Sterilization}

All medical equipment and personnel are subject to strict sterilization requirements in order to maintain a hygienic surgical environment. Several methods currently exist for the decontamination of equipment, including: autoclave; dry heat; chemical sterilization; and disinfectants [40]. As such, the final VACS must be able to withstand repeated exposure to sterilization processes without suffering undue effects that would compromise functionality. 


\subsection{Cautery Probe}

Cauterization probe design requirement aspects include items such as length, actuation, tool tip shape and probe insulation.

- Length: The length of the probe must equal the length of the VACS Endoscope plus the VACS Actuation Adaptor assembly plus the desired reach of the cautery unit from the distal tip of the VACS Endoscope.

- Actuation: The cauterization probe is required to have computer controlled mechanical extension and retraction. Therefore, the probe must be compatible with the actuation method selected.

- Tip: The distal end of the probe may adopt a variety of shapes, such as those depicted in Figure 4.3.

- Insulation: In addition, the probe must be insulated to avoid any harmful electrical currents passing to the actuator or endoscope.

\subsection{Machine Vision}

The machine vision and real-time image processing design requirements include items such as resolution and frame rate.

- Resolution: A high resolution video feed (i.e., a minimum of 1024 x 768), is critical to the accurate and successful identification of areas requiring cauterization. The current operating room video technology will satisfy this requirement. 
- Frame rate: Any real-time application will suffer from low frame rates. Therefore, attaining a suitable video frame rate of approximately $60 \mathrm{hz}$ should be a high priority.

\subsection{Voice Command}

The capability of controlling the VACS Surgical System using voice commands is critical to achieving the overall goal of VACS (i.e., to allow simultaneous control of hemostasis while addressing other essential surgical procedures). Therefore, training may be required to reach a high level of accuracy for voice recognition and voice commands.

\subsection{Robotic Positioning}

Traditional robotic positioning performance indicators, such as accuracy and repeatability do not apply to this design as the robotic positioner will be visually servoed (i.e., meaning that closed loop visual feedback will track and guide the end-effector to the target). Adequate robotic positioning resolution will be the key design requirement for the manipulator. A minimum resolution of $2 \mathrm{~mm}$ would be acceptable, considering cauterization often occurs from arcing between probe and tissue, making the precise positioning somewhat irrelevant. 


\section{Chapter 4}

\section{VACS Design Concept}


Current technology in the operating room provides medical personnel with digitally enhanced stereoscopic computer vision, robotically controlled precise surgical movement capabilities and voice-activated command of operating room devices. VACS represents an innovative consolidation of existing technologies with developing surgical robotics and machine vision advances, providing voice-operated and imageguided precise surgical movements.

In this chapter the conceptual design of VACS is presented in separate sections for each VACS component.

\subsection{VACS Endoscope}

The VACS endoscope design is an evolution of current laparoscopic equipment. While based upon traditional rigid endoscope construction, it incorporates a feature more commonly found in flexible endoscopes known as the instrument channel. The instrument channel is usually used to provide ventilation while investigating regions of the bronchus or esophagus, taking biopsies of questionable tissue and/or neutralizing ulcers through cauterization.

Figure 4.1 illustrates the VACS endoscope which includes channels for fiber-optic light sources, the optical lens, gas exchange and an additional channel providing

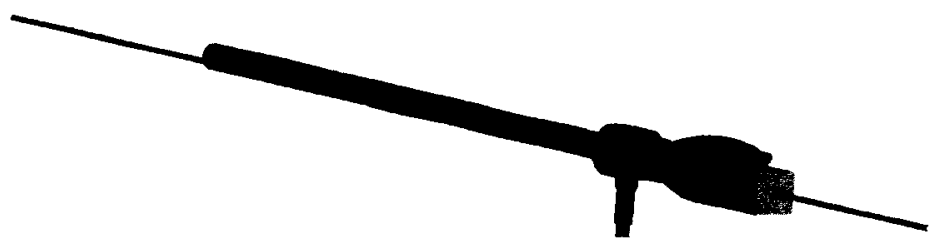

Figure 4.1: ProE model of VACS endoscope design. 
passage for the VACS cauterization probe, described in Section 4.3, where the VACS endoscope, actuation adaptor, HSI non-captive linear actuator and the cauterization probe are visible in the model.

Given that the basic size and functionality of the VACS endoscope does not deviate from current surgical scopes, it may be used to replace existing equipment. In so doing, VACS offers a range of additional surgical possibilities without compromising current technical capabilities. As a result, the VACS endoscope becomes a logical choice when replacing existing laparoscopic equipment. ${ }^{1}$

During traditional MIS, the laparoscope is connected, via a video adaptor, to a video receiver that is used to output the signal to monitors situated around the operating room. The typical adaptor receives the standard optical feed from the laparoscopic lens and converts it to one that may be passed through a fiber-optic cable to the receiver. The VACS endoscope design also includes a modified laparoscopic video adaptor (see Figure 4.2(b)). This modified adaptor is used to offset and reroute the video feed to provide sufficient space for the actuation mechanism which is mounted to the rear of the adaptor (i.e., though still aligned with the instrument channel of the laparoscope, discussed in Section 4.2).

\subsection{Actuation}

The VACS surgical system extends the cauterization probe beyond the distal tip of the VACS endoscope using computer controlled actuation. A non-captive linear actuator

\footnotetext{
${ }^{1}$ In the interests of accommodating hospital budgetary constraints the VACS system may be acquired and utilized in either its entirety or on an individual component basis (i.e., VACS endoscope, cautery probe, actuation, voice command software/hardware, machine vision software, surgical platform integration).
} 
(see Figure 4.2(a)) which can be controlled electronically, is mounted to the rear of the laparoscope. The linear actuator of choice is the Haydon Switch and Instrument Inc. 21000 Series - Size 8 Non-Captive Linear Actuator [41]. This specific model of stepper-motor style linear actuator, which measures less than $3 \mathrm{~cm}$ in length and $2 \mathrm{~cm}$ in height and width, will advance the cauterization probe in a lead screw fashion.

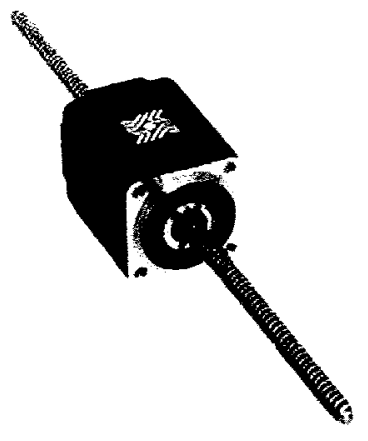

(a) HSI non-captive linear actuator [41].

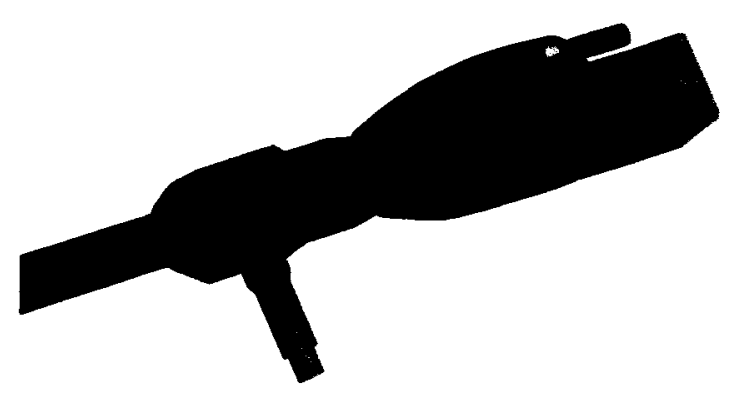

(b) ProE model of VACS Actuation Adaptor.

Figure 4.2: VACS endoscope actuator and assembly model.

\subsection{VACS Cauterization Probe}

The VACS cauterization probe is extended through the instrument channel of the VACS endoscope by the HSI non-captive linear actuator (i.e., as discussed in Section 4.2). The length of the probe must equal the length of the VACS endoscope and VACS actuation adaptor assembly plus the desired reach of the cautery unit from the distal tip of the VACS endoscope. The cauterization probe is actuated via the HSI non-captive linear actuator, requiring a portion of the cautery probe to be threaded to match that of the actuation system. The distal end of the probe may adopt a variety of shapes, such as those depicted in Figure 4.3. In addition, the probe 
must be insulated to avoid any harmful electrical currents passing to the actuator or endoscope.

The VACS endoscope has been designed to be compatible with two major types of surgical cautery: standard mono-polar electrocautery and argon beam cautery, thus helping to increase the practical adaptability of the VACS surgical system. Compatibility has been demonstrated by creating an ABS prototype VACS cauterization probe using a Dimension BST 3D printer.

\subsubsection{Electrocautery}

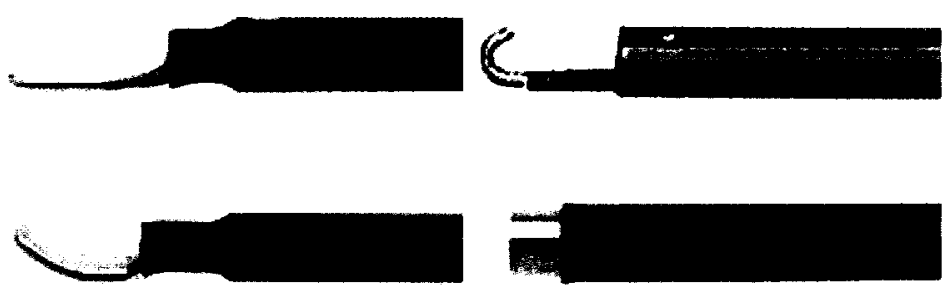

Figure 4.3: Laparoscopic cautery instruments [42].

Electrocauterization refers to the process of deliberately burning tissue with electricity. The burning heat is generated as high frequency electric current passes through the resistance of organic tissue. Therefore, the amount of heat delivered to the designated region is inversely proportional to the surface area of the electrode in contact with the tissue. This current is generated by an electrosurgical unit (ESU), which also connects to the patient through a grounding pad. The practice is frequently used to stop bleeding. Electrocauterization (or electrocautery) is considered a safe procedure that is routinely used in surgery to remove unwanted or harmful tissue. It can also be used to burn and/or seal blood vessels, which helps reduce or stop massive hemorrhaging. In order to cauterize effectively, the temperature of the tissue 
surrounding the probe must not exceed $100^{\circ} \mathrm{C}$ (i.e., the boiling temperature of water) which would cause the destruction of the cell through vaporization. Rather, it is more effective to modulate the current so as not to exceed $100^{\circ} \mathrm{C}$, whereby triggering a natural clotting response from the adjoining tissue and blood. Standard electrocautery temperatures are typically $60^{\circ} \mathrm{C}$ to $80^{\circ} \mathrm{C}$, however, anything beyond $55^{\circ} \mathrm{C}$ will cause cellular protein to denature and coagulate through clotting factors $[43]^{2}$.

\section{Cautery Experiment}

As discussed above, the cauterization heat is generated as resistance to the electric current passes through organic tissue, and as a result, heat delivered is inversely proportional to the surface area of the electrode in contact with the tissue (see Figure 4.4).

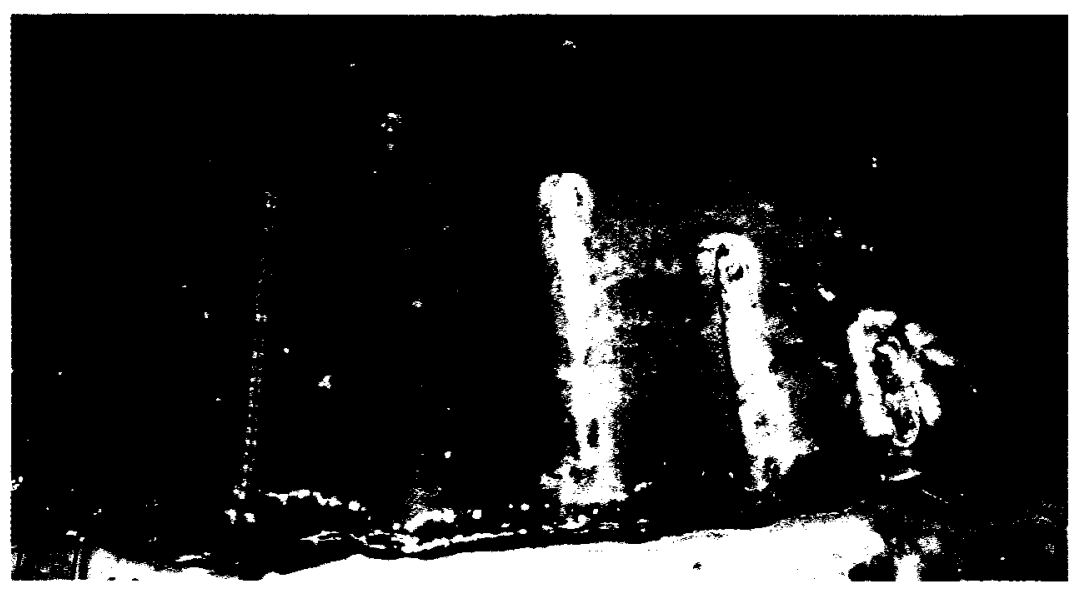

Figure 4.4: Liver cautery experimental results for varying cautery probe insertion lengths.

Figure 4.4 illustrates the amount of cauterization/desiccation of the tissue with

\footnotetext{
${ }^{2}$ Clotting Factors: A group of chemicals in the blood (factors I to XIII) that interact to form blood clots.
} 
respect to the penetration depth of the cautery probe. These experimental results were obtained using an electro surgical unit (ESU) at the Ottawa Skills and Simulation Centre. This particular piece of equipment had recently been replaced by a newer model in the operating room and was available for experimental procedures. The unit was set to standard surgical electrocautery output power of 30 watts (see Figure 4.5), as determined by first hand experience during five sperate laparoscopic surgical procedures. The cauterization probe was held in place at penetration depths $0.5,1.0,1.5$ and $2.0 \mathrm{in}$ and activated for a duration of $10 \mathrm{~s}$. Results indicate that optimal tissue desiccation occurs between $0-0.5$ in penetration. These results are conducive with the expected VACS design which does not require deep tissue penetration, rather only surface contact, to initiate a computer controlled cauterization sequence.

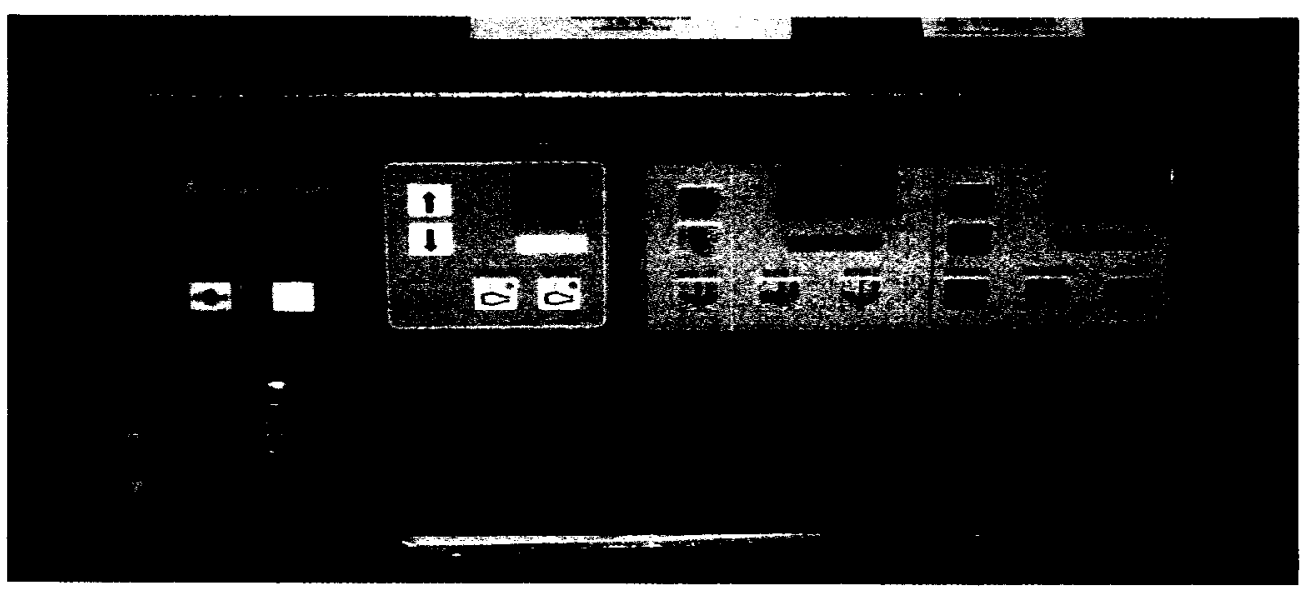

Figure 4.5: Valleylab ESU displaying coagulation power setting of 30 watts, as seen in central display. 


\subsubsection{Argon Beam Coagulator}

The Argon Beam Coagulator [44] is identical to a conventional ESU used to create an electrofulguration $\operatorname{arc}^{3}$. The difference is that the probe also delivers a 'purging' flow of inert argon gas during cauterization. The ionized argon gas creates a more uniform and homogenous form of cautery due to its lower resistance as compared to the typical $\mathrm{CO}_{2}$ used to insufflate ${ }^{4}$ the abdomen. When sufficient voltage is delivered through the gas stream, an argon plasma beam is formed thus cauterizing the tissue that it contacts. As a result, the probe itself does not need to directly contact the tissue given that electrical current tends to travel the path of least resistance. In the case of tissue that has not been adequately cauterized, the argon plasma is automatically diverted to non, or slightly-coagulated areas, resulting in a highly uniform cauterized surface.

\subsection{Surgical Platform}

The VACS design concept requires a robotic system capable of positioning the VACS endoscope using input from the VACS machine vision algorithm (see Section 4.7), in addition to potential voice commands, as discussed in Sections 4.5. Both the da Vinci Surgical System and the AESOP Endoscope Positioner (see Figure 4.6), satisfy this requirement and have been approved for use by the United States Food and Drug

\footnotetext{
${ }^{3}$ Electrofulguration: A form of treatment that destroys cells through incineration using an electrical current.

${ }^{4}$ Insufflate: To blow into; to fill any cavity or orifice of the body. Carbon dioxide $\mathrm{CO}_{2}$ gas is used to inflate the abdomen during laparoscopic surgery to help improve visualization.
} 


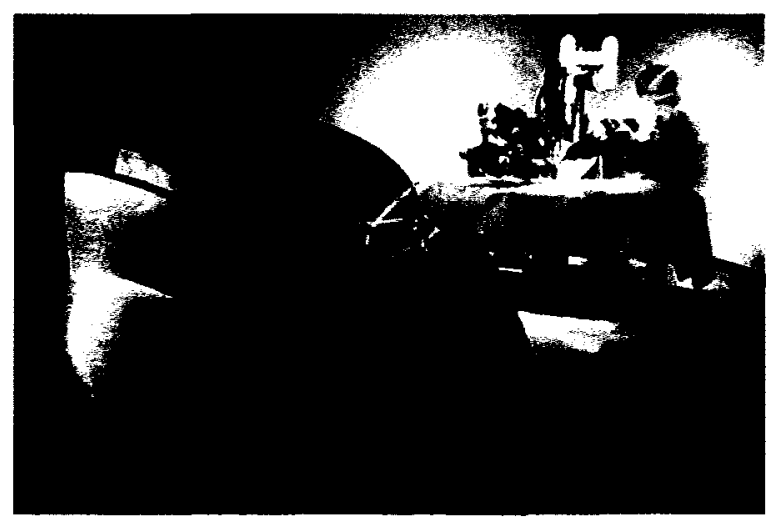

(a) da Vinci Surgical System [1].

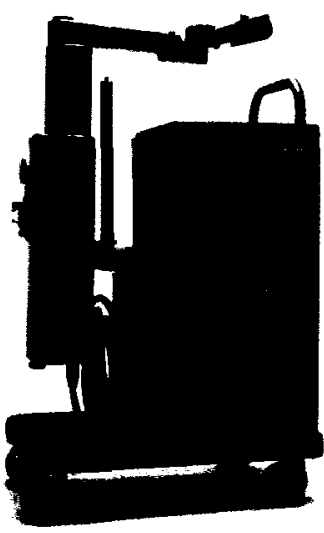

(b) $A E S O P[21]$.

Figure 4.6: Potential surgical platforms capable of manoeuvering the VACS with voice commands and meeting FDA approval.

Administration ${ }^{5}$.

\subsection{Voice Command}

The ability to control the VACS surgical system using voice commands is critical to achieving the overall goal of VACS (i.e., to allow simultaneous control of hemostasis while addressing other essential surgical procedures). To do so, the VACS machine vision algorithm will first identify potential cauterization sites before highlighting them on the operating room monitors. Afterwards, the surgeon may vocally initiate the cauterization procedure on a suitable site as interpreted by the system.

Currently there are a variety of surgical devices that include voice command capabilities $[1,21,35,36]$. Given that both the da Vinci Surgical System and the AESOP Endoscope Positioner offer voice interaction, both have been proposed, and

\footnotetext{
${ }^{5}$ The existence and development of the surgical technology described above demonstrates a favorable shift in the medical community's attitude towards the acceptance of surgical systems such as VACS.
} 


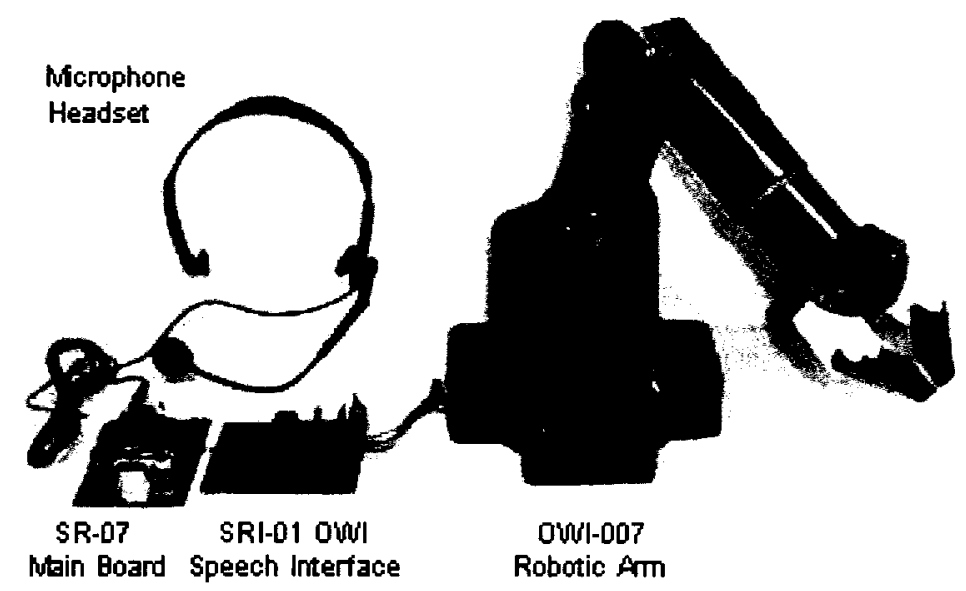

Figure 4.7: Speech Controlled Robotic Arm kit [45], including headset, voice recognition hardware, electrical activation hardware and robotic arm manipulator.

are strongly endorsed, as potential surgical platforms for the VACS (i.e., whereby further reinforcing their suitability for the VACS system implementation).

As for prototyping and proof-of-concept research, the "Speech Controlled Robotic Arm kit" offered by Image SI Inc. [45] includes the necessary hardware capable of satisfying the initial VACS design requirements. This kit is composed of the SR07 Speech Recognition Circuit (i.e., used to recognize and interpret commands) and the SRI-01 OWI Speech Interface Circuit (i.e., used to activate specific electrical circuits based on the original voice commands). Figure 4.7 shows these components in conjunction with a robotic arm manipulator.

\subsection{VACS Feedback System}

In order to produce autonomous artificial intelligence the VACS surgical system must be capable of determining the point at which the cautery probe has been sufficiently 
extended to contact the bleeding structure. This process is critical for achieving successful cauterization and for addressing obvious patient safety concerns.

To meet this requirement, a miniature load cell with thru-hole design manufactured by Honeywell [46], is incorporated into the design to return direct force measurement data during the extension of the probe. The load cell (see Figure 4.8(a)), is installed in the VACS actuation adaptor as shown in Figure 4.8(b), in order to measure the compressive force in between the adaptor and the actuator. In turn, the resistance force being experienced by the VACS cauterization probe will be transmitted through the probe to the actuator and will serve to diminish the compressive force acting on the load cell. By monitoring these fluctuations and calibrating the VACS software to identify a threshold indicative of tissue contact, the primary feedback mechanism is completed. Additionally, and as a means of further ensuring patient safety, the impedance and current being delivered for cauterization should be monitored to confirm a closed loop including the ESU, VACS cauterization probe, patient and grounding pad. Any discrepancies arising between the ESU power settings and readings from the rest of the closed loop system components would be immediately brought to the surgeon's attention.

Further description of the VACS control and feedback system will be discussed in Chapter 6 .

\subsection{VACS Machine Vision}

Image processing enables identification of bleeding structures that may then be cauterized by VACS at the surgeon's discretion.

Depending, for example, on the tuning of the image processing algorithm along 


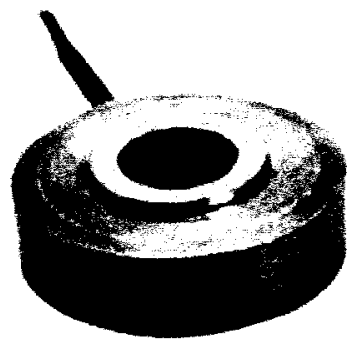

(a) Honeywell load cell with thru-hole [46].

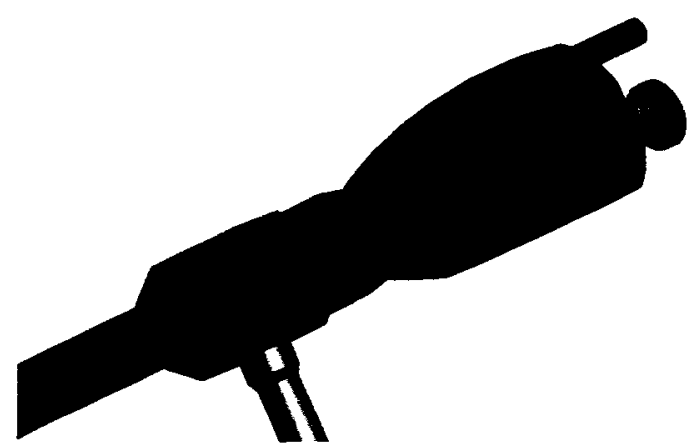

(b) ProE model of endoscope adaptor and load cell.

Figure 4.8: Load cell feedback components and ProE assembly model.

with the type and stage of a given surgical procedure, the image processing algorithm may identify multiple cauterization sites. These sites will be identified in real-time on the endoscopic monitor thus giving the surgeon the capability to choose to initiate the cauterization task on a particular region, using voice commands. Figure 1.1 shows examples of how the endoscopic image may be presented. In this visually autonomous system the surgical task would be performed under the surgeon's supervision but without his or her direct physical manipulation (i.e., thus allowing the surgical team to focus on other challenging surgical tasks).

VACS machine vision is discussed in Chapter 7. 


\section{Chapter 5}

\section{VACS Mechanical Design \& Rapid Prototyping}


Originally the concept of VACS was conceived as a robotic arm secured to the ceiling of an operating room; controlled by image processing and visual feedback in relation to an open surgical site. Upon discussion with surgical experts and time spent observing surgery, this concept seemed bulky, cumbersome and intrusive to be of any use to a surgical team. In addition, with significant advances in surgical robotics $[1,2,3]$, combined with the increasing popularity of minimally invasive surgery [4], demand for more sophisticated technologies capable of enhancing or extending current proficiencies $[5,6]$ is imminent. It is anticipated that the next generation of surgical robotics will be required to provide greater autonomous support [7]. As such, the conceptual design of VACS was shifted to facilitate MIS.

One of the several key concepts supporting MIS is the reduction in trauma to the patient due to incisions required for a given surgical procedure. Traditional open procedures require large invasive incisions in order to gain access and visualization of the necessary areas. Whereas, MIS principally aims to minimize the size of incision. Again, upon discussion with surgical experts and time spent attending MIS surgery, it was clear that adding an additional port for robotic access to the surgical area for cauterization was out of the question, due to the fact that current practices could accomplish the cauterization without any additional ports.

At this point the conceptual design evolved to the point where the cauterization tool was to be integrated with one of the existing ports. Logically the port accommodating the endoscope came to mind for this integration and the conceptual design of the VACS endoscope began to take shape.

The conceptual design of the VACS endoscope is based on the evolution and/or adaptation of a flexible bronchoscope. The key feature of the flexible bronchoscope, 


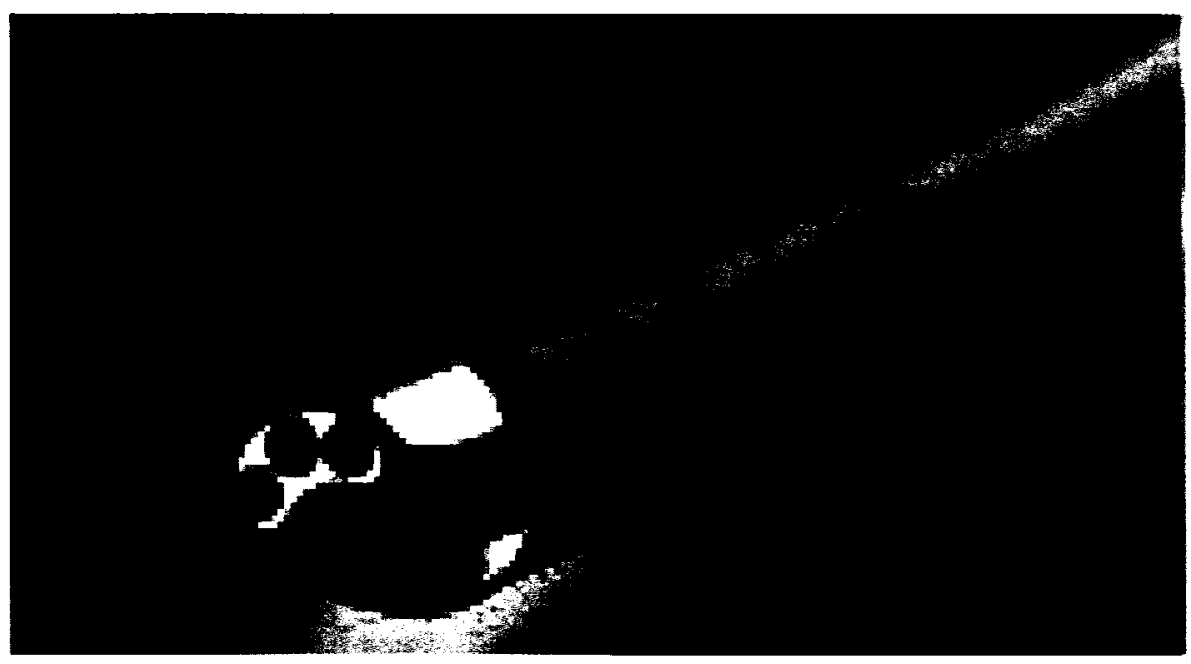

Figure 5.1: Distal tip of a flexible bronchoscope.

which to-date has not been duplicated in a rigid scopes is the instrument channel, shown in Figure 5.1.

The largest commonly used $\operatorname{trocar}^{1}$ for MIS can accommodate a $12 \mathrm{~mm}$ diameter instrument. Thus, the VACS endoscope was designed with an outer diameter of $12 \mathrm{~mm}$ to optimally use the $12 \mathrm{~mm}$ trocar's port space, while allowing for the current surgical practices to be maintained.

A 3D ProE model of the VACS endoscope was completed, as seen in Figure 5.2. Rapid prototyping of the ProE model was completed using the Dimension BST 3D printer. The Dimension BST 3D printer uses a stereolithography (.stl) file complied from the ProE model to print in ABS plastic, thus forming a solid model of the endoscope.

The rapid prototyping of the endoscope provided a tangible piece of equipment used to assist with the remainder of the design process. Including the sourcing of

\footnotetext{
${ }^{1}$ Trocar: the surgical tool passed through the body, used to allow easy exchange of endoscopic instruments during endoscopic surgery.
} 


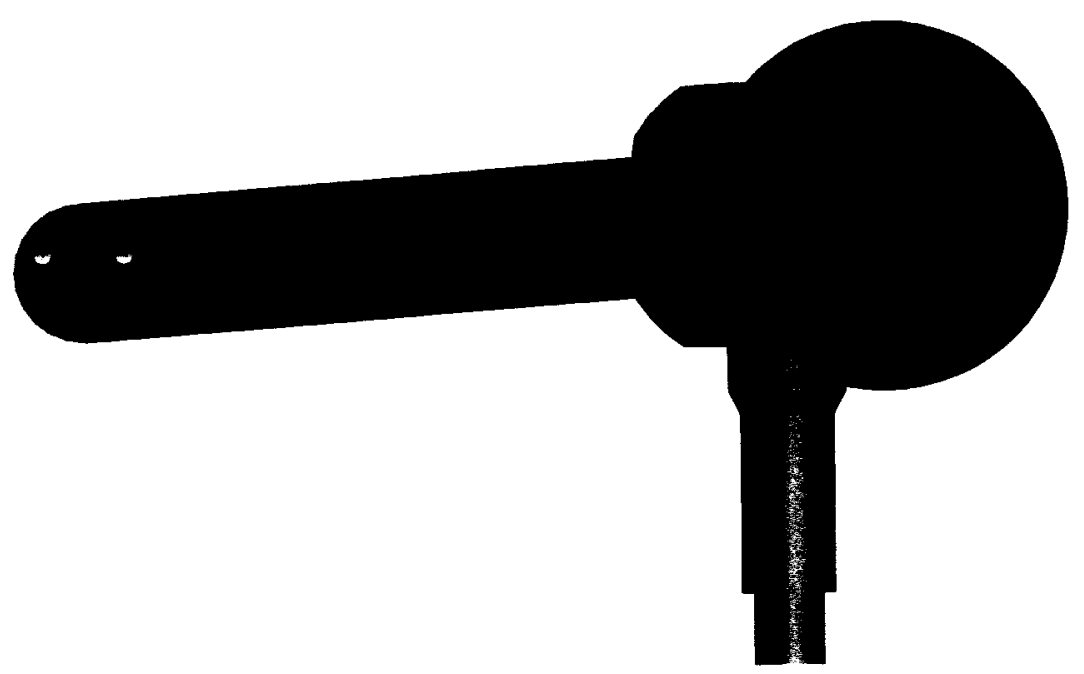

Figure 5.2: 3D ProE model of the VACS endoscope.

the linear actuator and load cell used in conjunction with the VACS endoscope, and eventually rapid prototype production of the actuation adaptor. 


\section{Chapter 6}

\section{VACS Control System}


The VACS control system is the software-based logic that forms the foundation for the machine vision and artificial intelligence (AI) control of surgical tasks and movements. As with most control systems, a computer is used to store the VACS's information about the work environment, technical data and surgical tasks. The control system also contains programs, data algorithms and logic analysis which enables the VACS to perform autonomously.

In this chapter several variations of surgical styles, as well as the VACS control system and artificial intelligence decision tree, are presented. The general architecture of a control system is represented in block diagram form.

\subsection{Surgical Modalities}

The modalities ${ }^{1}$ presented in Figure 6.1 represent the human-computer interaction of generally accepted surgical styles currently being performed. The figure is organized as follows: (a) open surgery; (b) minimally invasive surgery; (c) robotic surgery; (d) telerobotic surgery; (e) telemedicine or teleconsultation during surgery and (f) surgical simulation, where the type of information being transferred is denoted by (A)Audio; (M)Motion, Haptics or Force Feedback; (V)Vision; and (P)Positioning [47]. Of particular interest are surgical modalities (b), (c) and (d) because VACS is most applicable in these scenarios, due to the inclusion of voice activated robotics.

\footnotetext{
${ }^{1}$ In human-computer interaction, a modality refers to path of communication between the human and the computer, such as; audio, motion, vision or touch.
} 


\subsubsection{VACS Modality}

Figure 6.2 represents modality (c) from Figure 6.1 with the addition of a VACS style control over the endoscopic tool (i.e., the cautery tool) via voice recognition and AI.

\subsection{Functional Level MST}

MST or master-slave telemanipulator is the style of control system used in remote robotic feedback applications known as telerobotics. The MST system refers to the combination of five system components: operator, master, controller, slave and environment. The VACS control system design is comprised of these MST subcategories and illustrated in Figure 6.3.

As illustrated in Figure 6.3, the block diagram box labeled 'environment' represents the origin of the control system and cauterization task. The step by step interaction of the MST subcategories is as follows: the VACS endoscope captures the surgical environment; the VACS machine vision algorithm analyzes the endoscopic feed to identify potential areas of cauterization; the endoscopic video monitor presents the endoscopic feed combined with an overlay of the identified areas of potential cauterization; the surgeon then discriminates between the identified areas; through voice commands, the surgeon announces his intentions; voice recognition software interprets the voice commands and responds accordingly; if cauterization in requested for a certain area; the surgical robotic controller is activated; which positions the surgical robotic platform and VACS endoscope optimally for cauterization through feedback 
with the machine vision algorithm; subsequently, the stepper motor controller is initialized; which, activates the stepper motor to begin extending the cautery probe; once the cautery probe encounters environmental impedance in the form of tissue; the load cell incorporated into the VACS endoscope is triggered; provided the load cell registers a force greater than or equal to the tissue threshold, the stepper motor is deactivated; the cautery controller is then initialized; electrical current is delivered through the cautery probe tip; the tissue's electrical impedance, encountered by the current, produces the heat necessary for cauterization; the cautery probe ESU and internal multimeter monitor the electrical impedance; when the electrical current delivered drops to zero, cautery is complete (i.e., cauterization has desiccated the surrounding tissue and the tissue impedance has been correspondingly raised to a point where current will no longer complete a circuit at the designated power setting).

\subsection{Artificial Intelligence Decision Tree}

The decision level representation of the VACS control system (see Figure 6.4), illustrates the step by step sequence of inputs and decisions required for the preliminary VACS AI, represented in block diagram form with interaction as follows: the initial frame at time $t$ is captured from the endoscopic video feed; elementary colour segmentation is performed to identify feature points indicative of areas requiring cauterization (in the future, additional development of the image processing software may include colour edge detection, motion segmentation, background modelling or motion modelling); in each consecutive frame, the machine vision algorithm must determine if the feature point from the previous frame was tracked; if the point was not tracked, 
some type of occlusion handling, which is not yet developed, would ultimately manage the problem or re-initiate feature tracking with the current frame; if the feature point was tracked and based on whether the surgeon had requested cauterization, the system would continue to track the feature in subsequent frames; or robotic movement of the cautery probe would be initiated to bring the centroid of the area to be cauterized to the centre of the endoscope video feed; positioning of the endoscope and cautery probe is made possible through closed loop visual feedback between the endoscopic image and the machine vision algorithm's identification of the centroid belonging to the area to be cauterized; at this stage the area to be cauterized has been identified, tracked, discriminated by the surgeon, cautery has been requested, and the endoscope is positioned for cautery; the cautery probe is then extended until feedback from the load cell mounted in the VACS endoscope is triggered by tissue contact; the ESU then delivers the current required for cautery through the probe to the cauterization area; the cautery sequence is complete and halted when current no longer passes through the tissue due to the increased impedance effects that cautery has on tissue. 
(a)

(b)

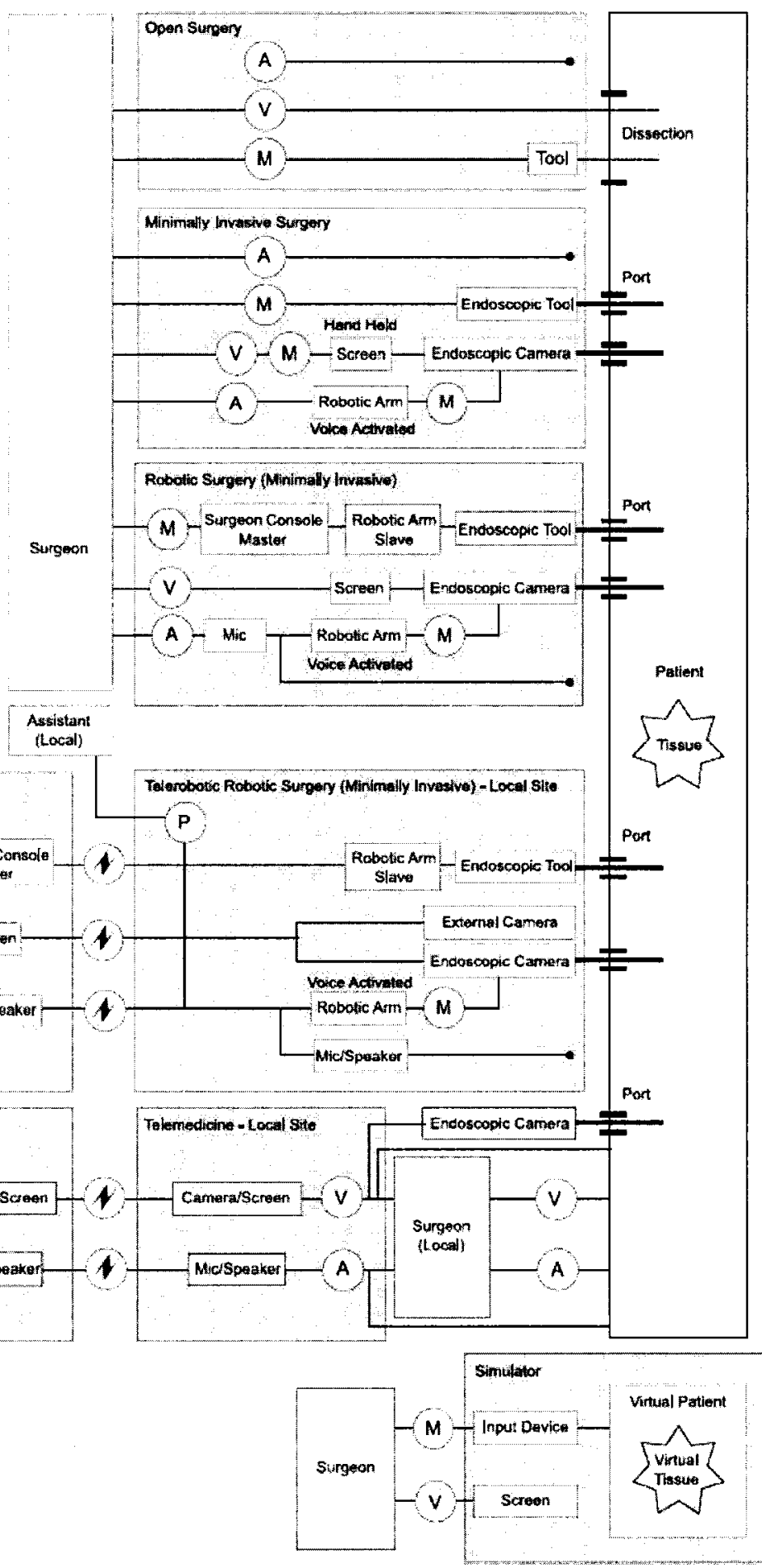

Figure 6.1: Modalities used in different configurations for performing surgery [47]. 


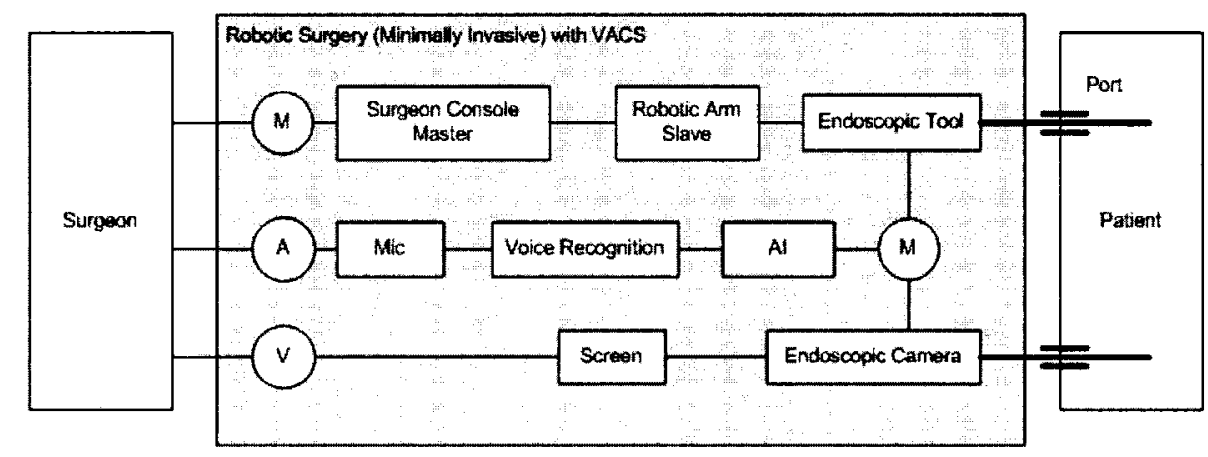

Figure 6.2: Block diagram modality representation of minimally invasive robotic surgery in conjunction with VACS control over surgical tool (i.e., cautery probe).

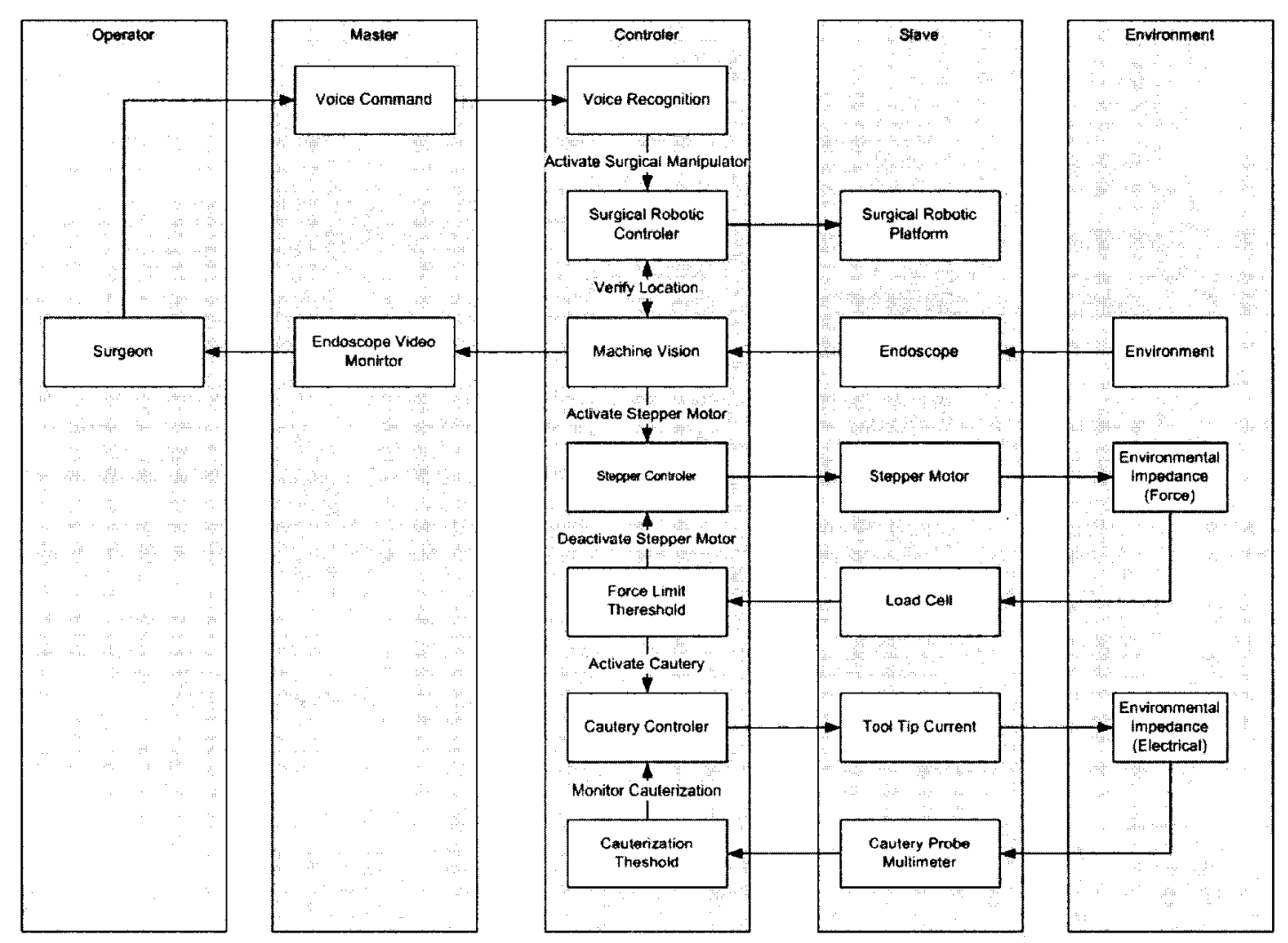

Figure 6.3: VACS control system expressed using MST subcategories. 


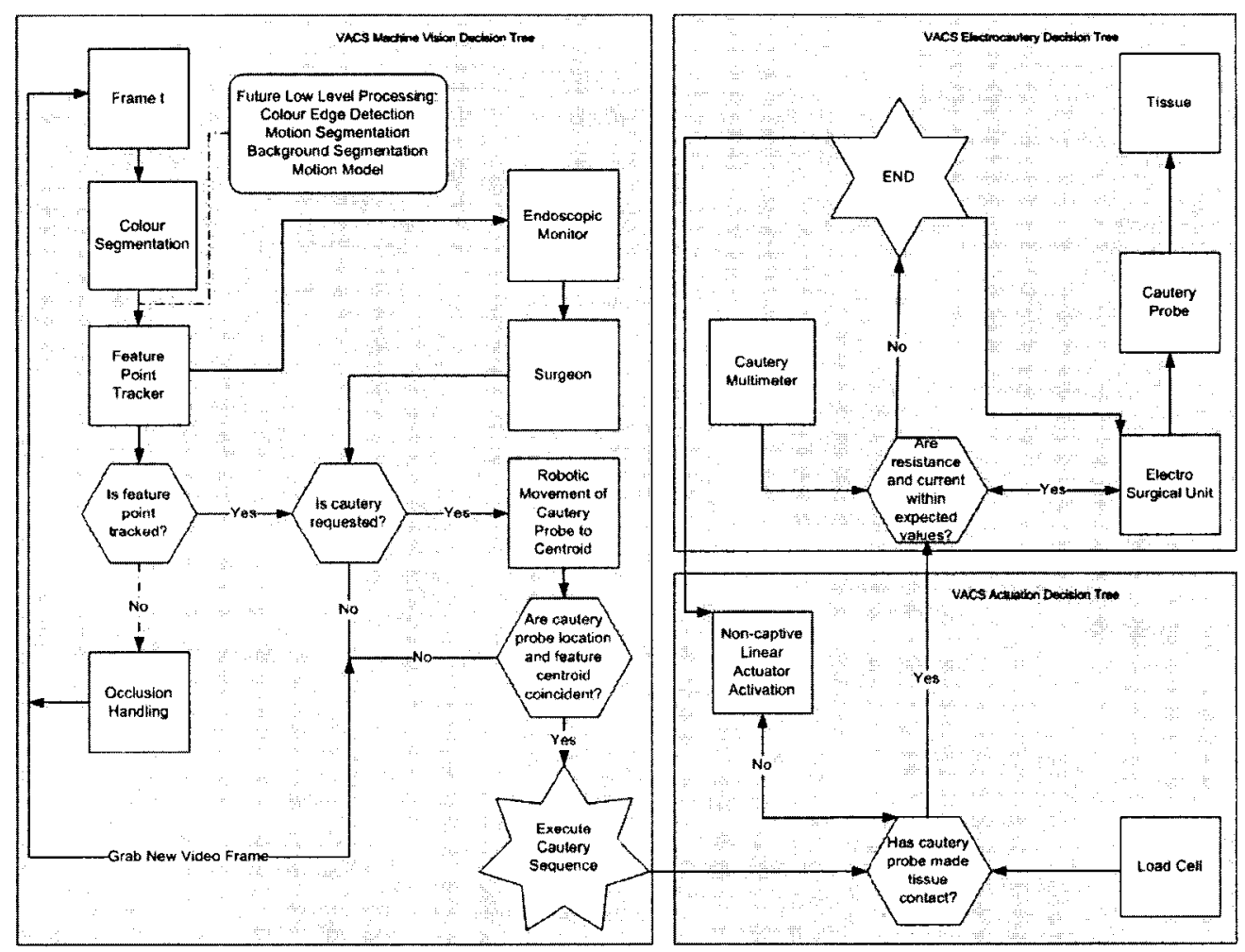

Figure 6.4: Artificial intelligence decision tree. 


\section{Chapter 7}

\section{Image Processing}


This chapter presents the machine vision ${ }^{1}$ research and analysis for real-time autonomous cauterization during Hand-Assisted Laparoscopic Liver Resection surgery[8]. This system implements digital image processing to identify regions requiring cauterization from a MIS laparoscopic video feed in real-time. Both a relative and absolute colour matching algorithm are implemented, and are capable of making identifications of cauterisable bleeding. The performance of the two algorithms are critically analyzed on the same video sequences. Additionally, a simple shape-based algorithm is implemented and the results are discussed. These preliminary algorithms are intended to be the basis for proof-of-concept, therefore, several areas are identified as requiring refinement and are discussed as future work.

In this chapter the image processing challenges, software algorithms and results are discussed.

\subsection{Problem Description}

Figure 7.1 exemplifies the challenges of feature recognition in surgical video. From an image processing standpoint, a generalized robust real-time solution is critical to successfully identifying and tracking features in the video feed. Multiple challenges are faced in both the video capture and video processing aspects.

\subsubsection{Real-time Medical Imaging}

Acquiring the video sequence adequate for image processing poses a significant challenge. Strict medical guidelines must be followed concerning any and all equipment

\footnotetext{
${ }^{1}$ Machine vision is a term used to define the process of integrating cameras and image processing into a system to provide increased autonomy.
} 


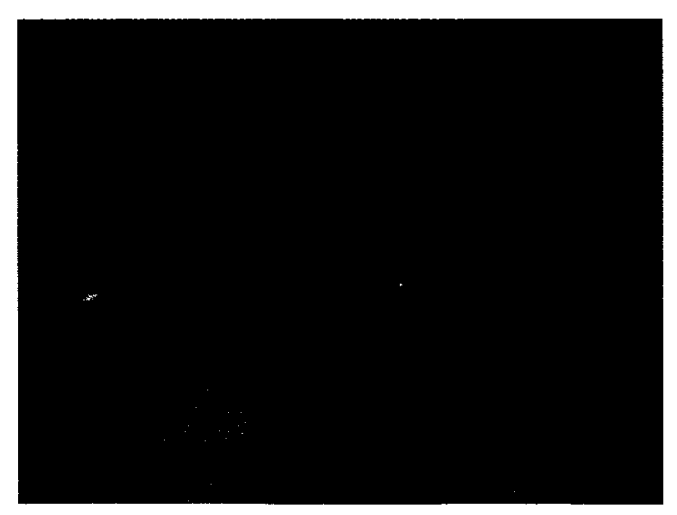

Figure 7.1: Video frame captured from hand-assisted laparoscopic liver resection [8].

located in the operating room. Additionally, the camera must be capable of acquiring consistently suitable video (i.e., resolution and frame rate).

\subsubsection{Moving Background}

Typically, motion estimation and object tracking can be facilitated using methods of background motion estimation and subtraction, as discussed in Section 2.1. Typically, background estimation is accomplished by generating a field of disparity vectors between features identified in successive frames. Areas with a uniform ficld of small vectors are assumed to be the background. In this case, however, the object and background are located on the same plane, therefore, no such method can be easily implemented.

\subsubsection{Moving camera}

The motion challenge is also compounded by the fact that the camera may not be stationary. In this particular case, the video sequence is captured from a laparoscopic camera being manipulated by hand. This significantly increases the amount and 
irregularity of motion compared to mounting the camera on a more stationary object or something that moves with more consistency (i.e., a robotic arm).

\subsubsection{Moving or Changing Object}

This appears to be a relatively basic aspect of the scenario, but accurately identifying an object in all subsequent frames is the challenging task addressed in all image processing and machine vision problems. The features in question are typically reddish circular areas indicating a bleeding blood vessel. However, their size and shape are not consistently uniform. Additionally, this component is the primary focus of the research.

\subsubsection{Clutter}

Clutter in image processing is defined as anything in the scene that is not the background or the region of interest. In this particular scenario, there are multiple objects throughout the laparoscopic scene that are not the object of interest (e.g., hands and various surgical instruments). This requires precise identification of unique features associated with the object to be identified.

\subsection{Suitable Algorithms}

Detection and tracking of abstract targets is a very complex problem and demands sophisticated solutions using pattern recognition and motion estimation methods. Although large amounts of research have been done, a generalized approach that satisfies all conditions has not been defined. However, there appear to be methods 
that are capable of satisfying individual aspects of the requirements. Perhaps a combination of multiple methods would allow the difficulties to be overcome. This proposed hybrid algorithm was examined primarily in the areas of object detection and object tracking $[24,26,28,29,31]$. These references help to form the basis of the implemented algorithm, including concepts such as:

- Feature detection

- Shape detection

- Colour histogram

- Background estimation

- Object learning

Of particular interest are techniques focused on feature detection using colour and shape characteristics. In turn, this will be the basis of the research and algorithm presented in the remainder of this chapter. Background estimation is not applicable given that both the objects of interest and background lie in the same plane, and areas of both tend to move uniformly. In addition, object learning would not be suitable to implement due to time and object variability constraints.

\subsection{Implemented Algorithms}

The hybrid approach was implemented in two parts: the colour matching phase and the shape determining phase. These phases are meant to first detect and combine objects of interest using the color algorithm and then to eliminate false positive matches by shape comparison. 


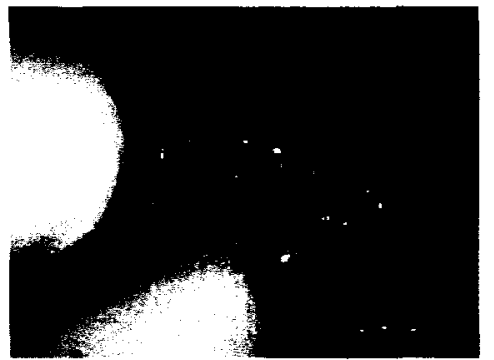

(a) RGB input, img(:,:,:) [8].

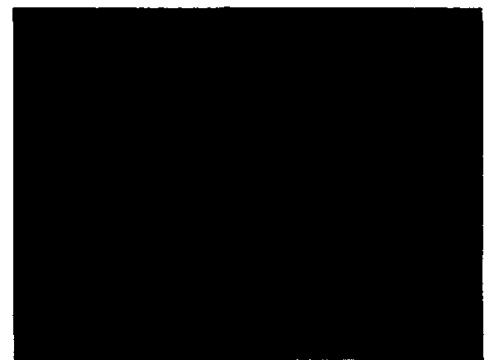

(b) Greyscale identification, (c) redimg.

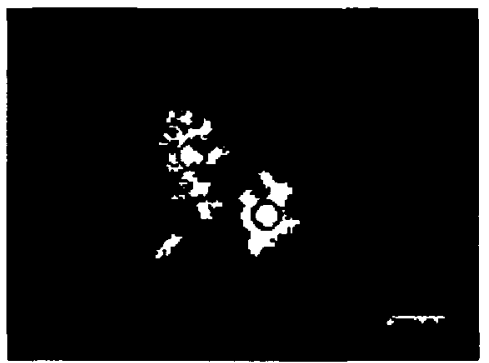

(c) Black and white threshold re sult, bwimg.

Figure 7.2: Relative frame processing sequence.

\subsubsection{Colour matching}

Colour is the principle identifying feature of this implementation. The reddish colour of blood is very distinct in the operating environment, helping significantly to identify areas requiring cauterization. As a point of interest, both relative and absolute colour matching algorithms were developed in MATLAB with the assistance of the Video and Image Processing Toolbox [48]. The full length MATLAB relative and absolute coded algorithms can be found in Appendices A and B respectively.

\section{Relative colour matching}

The unique aspect to this approach is the insensitivity to slight illumination or intensity shifts. The algorithm is designed to identify pixels having the highest reddish appearance based on the ratio of RGB colour channels. The primary logic behind the relative colour approach was implemented in MATLAB as follows,

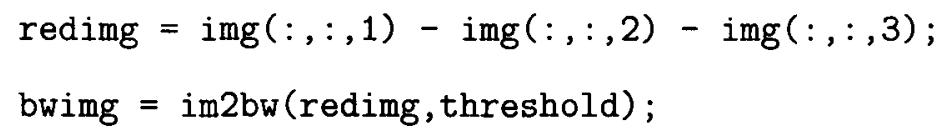


where $\operatorname{img}(:,:,:)$ is the input frame in the RGB colour space (see Figure 7.2(a)). The output image bwimg (see Figure 7.2(c)) is the thresholded representation of the green and blue channels subtracted from the red channel (see Figure 7.2(b)). As such, if the intensity of the image were to drop due to a shift in lighting conditions (e.g., debris covering or interfering with the fiber optic light source), the values of all pixel channels would drop but the algorithm would retain the ability to identify the strongest reddish pixel.

\section{Absolute colour matching}

This algorithm is highly flexible and allows all RGB intensity and threshold values to be tuned separately. The absolute colour approach was implemented pixel-wise (two for-loops) in MATLAB as follows,

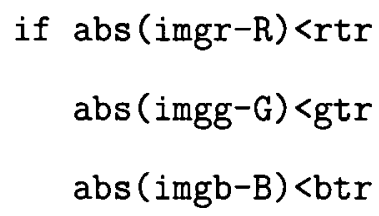

where imgr, imgg, imgb represent the input image pixel colour intensity, R, G, B are the specific colour intensities to be identified, and rtr,gtr, btr are the acceptance thresholds for pixel intensity variations.

The original input video frame (see Figure 7.3(a)), shows the surgery as seen from the laparoscope. The digital image processing algorithm described above produces the black and white image by making pixels that satisfy the criteria white and setting the rest of the image to black (see Figure 7.3(b)). Similar to the relative approach, this black and white image can be used to guide a robotic end-effector to a region of interest based on the location of the region's centroid. 


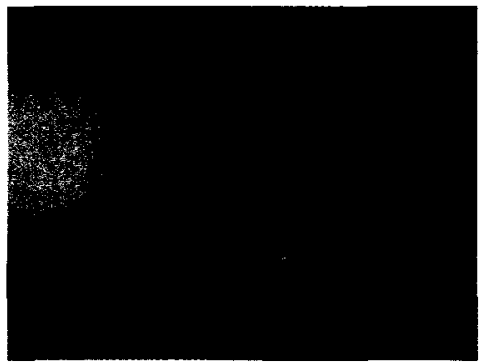

(a) RGB input [8].

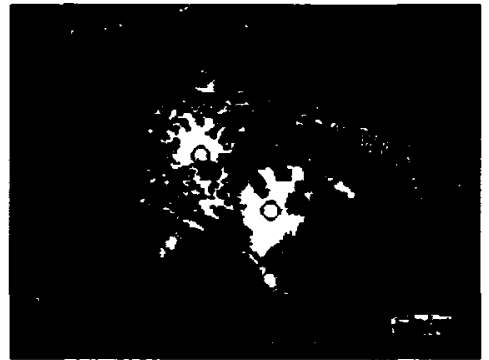

(b) Black and white threshold result.

Figure 7.3: Absolute frame processing sequence.

\subsubsection{Shape matching}

In addition to colour, shape can be used as an added method of narrowing the number of potential targets. Analysis of both the colour matching algorithms indicates the potential for false positive matches. Commonly, bleeding in the surgical region appears initially as an enlarging reddish dot or circle. With this in mind, an additional phase was added to the approach to help produce this identification and reduce the number of false targets.

\section{Roundness metric}

In this particular application, an object's circularity or roundness is considered very important because it provided additional criteria with which to analyze the image. The roundness metric is a simple formula used to estimate an object's circularity based on area and perimeter, and is calculated as follows,

$$
\text { metric }=4 * \text { pi*area } / \text { perimeter }{ }^{` 2} \text {; }
$$

where the area and perimeter values are derived, as approximations, using MATLAB and the Image Processing Toolbox functions. Perfectly circular objects have a metric 
of 1 , while all other objects have a value $<1$, as such, a threshold applied to the objects' roundness metrics will eliminate less round objects as possible targets. Based on this metric, certain reddish regions generated by shadows or irregular objects could be eliminated due to their lack of roundness. Using the roundness threshold in conjunction with the colour matching algorithms, helps to eliminate false positive results.

For this particular analysis, it was only suitable to implement the roundness metric algorithm on the video sequence seen in Figure 7.3 (i.e., primarily due to the fact that this sequence shows multiple false positive results (see Figure 7.3(b)), while the video sequence seen in Figure 7.2 is free of this type of error). However, the video resolution was poor, and often after analysis, objects of interest were only reported as two or three pixels. This inhibited the accuracy of the metric algorithm rendering it useless in a practical application.

\subsection{Performance Assessment}

The relative colour algorithm performs more accurately and is more computationally efficient compared to the absolute algorithm, only requiring two lines of code (1920000 operations for a $1600 \times 1200$ resolution frame) rather than two for-loops to call each pixel and check all RGB channel values (5760000 operations for a 1600x1200 resolution frame). However, the absolute algorithm is inherently more flexible in design, as the RGB and threshold values can be modified directly. The relative algorithm is not without the possibility of adjustment. However, both the ratio between RGB values could be modified in the subtraction phase and the thresholding value could also be adjusted to gain some added flexibility from the relative algorithm. 
The results of quantitative analysis of the 39 frame video sequence from the endoscopic feed of the liver resection reveal the following; The relative colour algorithm captured 56 suitable cauterization sites and identified 41 false positive sites. This gives a performance index (hits/misses) of 1.36 . Whereas, the absolute algorithm results from the same video sequence gave 48 hits on cauterization sites and 68 misses. This resulted in a performance index of 0.70 . The comparison of performance indices clearly identifies the relative colour algorithm as superior in this comparison. Subsequent testing is necessary to determine if this result is true in general. 


\section{Chapter 8}

\section{Discussion \& Conclusions}


The issues identified in the conceptual design of VACS underscore the considerable challenges involved in the development of advanced medical technologies. At the same time, VACS serves to represent a valuable interdisciplinary bridge between multiple engineering and surgical communities. As the literature review indicates, VACS is a unique visually servoed surgical robotic system. Should the current trend in autonomous surgical support continue as expected, it is not unreasonable to anticipate that future generations of surgical robotic technology will embody characteristics of VACS. The intense support and interest in this project suggests that the major objective of VACS has already been achieved. Namely, to demonstrate that technological innovations exist to advance the frontiers of surgical technology.

The feasibility of a visually semi-autonomous surgical assistant capable of performing cautery was established by discussions with surgical experts and research into current surgical robotic technologies. The design and fabrication of a prototype endoscope compatible with current surgical demands, as well as those of the VACS, was completed and discussed in detail in Chapters 4 and 5 of this thesis. In addition to establishing the feasibility of VACS, discussions with surgical experts lead to the identification of the surgical application for which VACS is both capable and feasible. A suitable experimental surgical niche in which VACS can be tested was also identified through discussion with the medical community. Most importantly, from a medical policy/legislation point of view, the consultations with medical professionals provided the opportunity to deduce the acceptability and acceptable limits of autonomy for a semi-autonomous cautery surgical assistant.

Component-specific design requirements include the following:

- MIS Entry Method: The VACS endoscope provides an entry method that 
includes the instrument channel for the cauterization probe. Thus, no additional incisions are required.

- Minimal Deviation from Current Surgical Practices: The VACS endoscope design is based upon current endoscopic technology. As such, it can be used as a substitute in the same capacity as current endoscopes or in conjunction with other components of VACS in order to provide more autonomous support. At the same time, the system does not interfere with or create any obstacles for current surgical practices. Consequently, in the presence of VACS and all of its components, a surgeon would be able to revert to standard practice by simply ignoring the system.

- Component and Patient-Safe Electrocautery: The insulated cauterization probe prevents electrical discharge through the VACS endoscope to the patient or any other conductor. This ensures that actuation, feedback, and optical components will not be affected by electrical shock. Moreover, it guarantees that the cautery charge will not be inadvertently discharged through the endoscope into any non-intended tissue with which it may make contact.

- Machine Vision: The image processing algorithm is capable of identifying potential locations requiring cauterization. Combining this feature with the robotic capabilities of a $d a$ Vinci or $A E S O P$ system provides simulated artificial intelligence or "de facto" machine vision.

- Surgeon commanded control: In the interests of enhancing surgical proficiency while accommodating all necessary safety concerns, VACS intends to identify and present potential bleeding locations to the surgeon (i.e., via an 
overlay on the surgical video monitors). At this point, and if desired, the surgeon can choose to initiate the cauterization sequence on any of the identified areas. The cauterization task would then be performed autonomously by the VACS. This design provides the operating surgeon with simultaneous control over hemostasis while freeing him or her to concentrate on other aspects of the procedure by transferring the physical steps to the robotic manipulator, thus enhancing the surgeon's capabilities.

- Electrocautery Feedback: In order to perform and reset for repeated cauterization tasks - while meeting obvious safety concerns - VACS requires a feedback mechanism capable of ensuring successful manipulation of the cautery probe, initiation and completion of the electrocautery task. This objective is achieved through the combination of a force feedback load cell and monitoring of the electrical current being delivered for cauterization. 


\section{Chapter 9}

\section{Recommendations \& Future Work}


As a significant advance towards proof-of-concept, this research should prove to be a valuable contribution to the future of surgical robotics. At the same time there remains a need for continued research and refinements leading to the creation of a physical prototype. Areas of research should include the following.

- $30^{0}$ Endoscope Implementation: Endoscope manufacturers often offer scopes with lens angles that deviate from $0^{0}$, meaning that the line of sight is angled in relation to the endoscopic shaft axis. This type of design presents challenges considering that the cautery probe is also extended through the instrument channel running parallel to the shaft axis. The design of an instrument channel or cautery probe capable of any orientation from the distal tip of the endoscope would increase the versatility of VACS

- Reduce Endoscope Shaft Diameter to Increase Versatility: Reducing the VACS endoscope shaft diameter would extend the use of VACS to other surgical procedures, where the size of the trocar may not accommodate the current $12 m m$ design.

- Resolution: Unfortunately, the video publicly available online was limited to a resolution of $320 \times 240$. As mentioned, the roundness metric's accuracy was significantly reduced due to the low resolution. Therefore, by increasing the video resolution, more accurate pixel-wise detail would be introduced. Such an upgrade would help contribute to both improved colour and shape-based results. This improvement would naturally be realized when real-time surgical video is analyzed from the endoscopic feed as the digital video resolution is likely $1024 \times 768$ if not $1600 \times 1200$ in most operating rooms. 
- Optical flow: Clutter in the video frame and occlusion of the surgical site could be overcome by implementing an optical flow algorithm to track and eliminate the surgeon's hand movement (e.g., or other objects occluding the scene and potentially being mis-identified as bleeding areas), thus allowing attention to be focussed on relatively stationary portions of the image.

- Implement probabilistic techniques: Bayesian merge $[49,50]$ and DempsterShafer theory of evidence $[51,52]$ are both techniques that would help to identify and track objects in the video sequence. They provide a method to store past information concerning regions of interest, thus allowing future decisions to be based on history and probability rather than simply the information currently available.

- Maintain high frame rate: Any real-time application will suffer from low frame rates. Therefore, while future work is being considered and implemented, maintaining a suitable frame rate should be a high priority.

- Color mapping: While RGB is the most traditional color mapping, alternatives exist that may prove to facilitate the identification of areas requiring cauterization (e.g., LAB, HLS, HSV, etc.).

- Vision alternatives: Although optical sensing is sufficient for humans to perform surgery, this should not limit the possibilities of machine vision. Installing a unique filter on the camera or replacing the camera with some type of scanning system to identify high traces of iron, (i.e., a substance found in the hemoglobin of blood) could increase the image processing accuracy. Alternate or even nonconventional methods may prove more effective given this unique scenario. 


\section{Bibliography}

[1] G. Guthart and K Salisbury. "The Intuitive Telesurgery System: Overview and Appliation.". IEEE International Conference on Robotics and Automation, pages $618-621,2000$.

[2] J. Marescaux, J. Leroy, M. Ganger, F. Rubino, D. Mutter, M. Vix, S Butner, and M. K. Smith. "Transatlantic robot-assisted telesurgery." Nat. Mag., 413:379380, 2001.

[3] Engaget. "Robot Surgeon Performs World's First Unassisted Operation". Engadget, http://www.physorg. com/news67222790.html, May 2006.

[4] PRNewswire. "Growing Popularity of Minimally Invasive Surgery Drives Growth in Coronary Stents Market". http://www. prnewswire.co.uk/cgi/news/ release? $i d=161908,2006$.

[5] CSTAR. "Canadian Surgical Technology \& Advanced Robotics". http://www. c-star. ca/about. aspx, 2006.

[6] EndoVia. "EndoVia Medical". http:// endovia. millersystems. com/ about_us/, 2006. 
[7] Gary S. Rogers and Caroline G. L. Cao. "Computer-Enhanced Instruments: The Next Generation of Surgical Robots". http://ase. tufts. edu/mechanical/ EREL/web/Next\%20Generation\%20of\%20Surgical\%20Robots. pdf , 2006.

[8] Medline Plus. "Videos of Surgical Procedures", http://www.nlm.nih.gov/ medlineplus/surgeryvideos.html, 2005.

[9] ACR RSNA. "Computed Tomography (CT)". http://www. radiologyinfo. org/content/, 2005.

[10] Cambridge Surgical Hepatobiliary and Pancreas Service. "Patient Agreement to Investigation or Treatment - Liver Resection". Addenbrooke's Hosptical - NHS, 2004.

[11] T Clark Gamblin and David A Geller. "Laparoscopic Liver Resection 2005 Update". Liver Cancer Center, University of Pittsburgh School of Medicine, 2005 .

[12] MD Yuman Fong, MD William Jarnagin, MD Kevin C. Conlon, MD Ronald DeMatteo, RN Ellie Dougherty, and MD Leslie H. Blumgart. "Hand-Assisted Laparoscopic Liver Resection". Archives of Surgery, 135:854-859, July 2000.

[13] Department of Surgery Univeristy of Southern California. "Laparoscopic Liver Resection". Center for Pancreatic and Biliary Diseases, 2006.

[14] F.A.C.S. Leonidas G. Koniaris, M.D. "Approaching Liver Tumors and the Phenomenon of Liver Regeneration". University of Miami Hospital and Clinics, Jackson Memorial Hospital, 2006. 
[15] M.D. Tse-Ling Fong and Ph.D. Leslie J. Schoenfield, M.D. "Liver Resection". MedicineNet.com, 2005.

[16] Michalopoulos GK and DeFrances MC. "Liver regeneration". Science, 276:60, 1997.

[17] USC Liver Transplant Program and Center for Liver Disease. "Liver Surgery". http: //www. surgery. usc. edu/divisions/hep/liversurgery. html, 2005.

[18] USC Liver Transplant Program and Center for Liver Disease. "Bloodless Surgery". http://www. surgery.usc. edu/divisions/hep/ bloodlesssurgery. html, 2005.

[19] The University Hospital University of Medicine \& Dentistry of New Jersey. "Bloodless Surgery and Medicine". http://www. theuniversityhospital. com/bloodless/, 2005.

[20] Graduate Hospital Tenet Pensylvainia. "The Center for Bloodless Medicine \& Surgery". http://www.graduatehospital.com/ CWSContent/graduatehospital/ourServices/medicalservices/ centerBloodlessMedicineSurgery. htm, 2005.

[21] T. Broderick, K. Russell, C. Doarn, and R. Merrell. "A novel method for visuallizing the open surgical field". Journal of Laparoscopic and Endoscopic Advanced Surgical Technology, 12(4):297-302, 2002.

[22] FDA. "US Food and Drug Administration". http://www. fda. gov/, 2006. 
[23] Ramesh Jain, Rangachar Kasturi, and Brian G. Schunck. "Machine Vision". McGraw-Hill and MIT Press, 1995.

[24] Alireza Behrad, Ali Shahrokni, Seyed Ahmad Motamedi, and Kurosh Madani. "A Robust Vision-based Moving Target Detection and Tracking System". Electrical Engineering Department AMIRKABIR University of Technology, Intelligence in Instrumentation and Systems Lab.(I2S) SENART Institute of Technology - University PARIS XII, 2001.

[25] Bryan S. Morse. "Region Based Segmentation". Brigham Young University, http://homepages.inf.ed.ac.uk/rbf/CVonline/LOCAL_COPIES/MORSE/ region.pdf, 2000.

[26] Paul Withagen, Klamer Schutt, and Frans Groen. "Likelihood-based Object Detection and Object Tracking Using Color Histograms and EM". IEEE ICIP, TNO Physics and Electronics Laboratory, IAS group, University of Amsterdam, 2002.

[27] Steven Ruzin. "Image Processing III Binary operations". University of California, Berkeley, http://microscopy.berkeley.edu/courses/dib/sections/ 04IPIII/binary.html, 2004.

[28] Shree K. Nayar, Sameer A. Nene, and Hiroshi Murase. "Subspace Methods for Robot Vision". Department of Computer Science, Columbia University, New York, February, 1995. 
[29] Michail Isard and Andrew Blake. "CONDENSATION-Conditional Density Propagation for Visual Tracking". International Journal of Computer Vision Department of Engineering Science, University of Oxford, Kluwer Academic Publishers, 1998.

[30] Andy Salter. "B-Spline curves". Imperial College, http://www.doc.ic.ac.uk/ $\sim \mathrm{dfg} /$ AndysSplineTutorial/BSplines.html, 2006.

[31] Andrea Cavallaro, Olivier Steiger, and Touradj Ebrahimi. "Tracking video objects in cluttered background". IEEE TRANSACTIONS ON CIRCUITS AND SYSTEMS FOR VIDEO TECHNOLOGY, 2004.

[32] Andrea Cavallaro and Touradj Ebrahimi. "Video Object Extraction Based on Adaptive Background and Statistical Change Detection". In Proceddings of SPIE Electronic Imagin - Visual Communications and Image Processing, pages 465475, 2001.

[33] TRUEFORCE. "Intuitive Surgical and Computer Motion Merge". http: // trueforce. com/News/ComputerMotionNews/Computermotion20030307. htm, March 7, 2003.

[34] TRUEFORCE. "Industry Profile: Computer Motion Inc. - Hermes". http://trueforce. com/Medical_Robotics/Medical_Robotics_ Companies/hermes. htm, 2006.

[35] Mease Countryside Hospital. "Mease Countryside Hospital Launches VoiceControlled Operating Room". http://www. measehospitals. com/11012. cfm, 2003. 
[36] Smith \& Nephew Endoscopy. "Smith \& Nephew Endoscopy's Condor Control System". http://www. smith-nephew. com/news/item. jsp? $i d=295,2006$.

[37] Gregory Tholey, Anand Pillarisetti, William Green, and Jaydev P. Desai. "Design, Development, and Testing of an Automated Laparoscopic Grasper with 3-D Force Measurement Capability". Program for Robotics, Intelligent Sensing, and Mechatronics (PRISM) Laboratory, Drexel University, 2004.

[38] Jacob Rosen, Blake Hannaford, Mark P. MacFarlane, and Mika N. Sinanan. "Force Controlled and Teleoperated Endoscopic Grasper for Minimally Invasive Surgery Experimental Performance Evaluation". IEEE TRANSACTIONS ON BIOMEDICAL ENGINEERING, VOL. 46(NO. 10), OCTOBER 1999.

[39] Victor F. Munoz, J. Gomez de Gabriel, J. Fernandez-Lozano, I. Garcia-Morales, R. Molina-Mesa, C. Perezdel-Pulgar, J. Seron-Barba, and M. Azouaghe. "Design and control of a robotic assistant for laparoscopic surgery". Instituto de Automatica y Robotica Avanzada de Andalucia. Universidad de Malaga Severo Ochoa, 2001.

[40] University of Medicine and Dentistry of New Jersey. "EOHSS FACTSHEET Decontamination and Disinfection". http://www2. umdnj. edu/eohssweb/ publications/disinfection.pdf, 2006.

[41] Haydon Switch and Instrument Inc. "HSI Linear Actuators - 21000 Series - Size 8 Linear Actuator". http://www. hsimotors. com/hybrid/ size8-hybrid-linear-actuator_noncaptive. $h t m, 2005$. 
[42] Valleylab. "Laparoscopic Instrumentation". http://www. valleylab. com/ product/es/lap/index. html, 2006.

[43] D. Mutter. "Basic Principles: Electrocautery and High-Frequency Currents in Surgery". http://www. websurg. com/tableofcontents/index. php? spec $=5,2001$.

[44] Gregory T. Absten. "Practical Electrosurgery For Clinicians". http://www. lasertraining. org/electrosurgery. htm, 2002.

[45] Image SI Inc. "OWI Robotic Arm and Interfaces". http://www. imagesco. com/catalog/RoboticArm/RoboticArm01. html\# speech, 2006.

[46] Sensotec. "Honeywell Load Cell". http://www. sensotec. com/ $p d f / d . p d f$, 2006.

[47] J. Speich and Jacob Rosen. "Medical Robotics". Encyclopedia of Biomaterials and Biomedical Engineering, pages 983-993, 2004.

[48] The MathWorks. "Image Processing Blockset 1.1". MATLAB, http://www . mathworks . com/access/helpdesk/help/toolbox/images/images . shtml, 2005 .

[49] Pablo Alvarado and Axel Berner. "Segmentation of Hand-Held Skin-Colored Objects Using Bayesian Belief Networks". Department of Technical Computer Science, RWTH Aachen, Germany, http://www.techinfo.rwth-aachen.de/ Veroeffentlichungen/V003_2002.pdf, 2002.

[50] Santiago Venegas, Gianluca Antonini, Jean-Philippe Thiran, and Michel Bierlaire. "Bayesian Integration of a Discrete Choice Pedestrian Behavioral Model 
and Image Correlation Techniques for Automatic Multi Object Tracking". Swiss Federal Institute of Technology (EPFL) Signal Processing Institute (ITS) and Operation Research, http://lts1pc19.epfl.ch/repository/Venegas2004_773. pdf, 2004.

[51] Azzedine Bendjebbour, Yves Delignon, Laurent Fouque, Vincent Samson, and Wojciech Pieczynski. "Multisensor Image Segmentation Using DempsterShafer Fusion in Markov Fields Context". IEEE TRANSACTIONS ON GEOSCIENCE AND REMOTE SENSING, VOL. 39, AUGUST 2001.

[52] Huadong Wu. "Sensor Data Fusion for Context-Aware Computing Using Dempster-Shafer Theory". The Robotics Institute Carnegie Mellon University, http://www.cs.cmu. edu/ whd/publications/whd-thesis.pdf, 2003. 


\section{Appendix A}

\section{Image Processing Algorithm -}

\section{Relative}




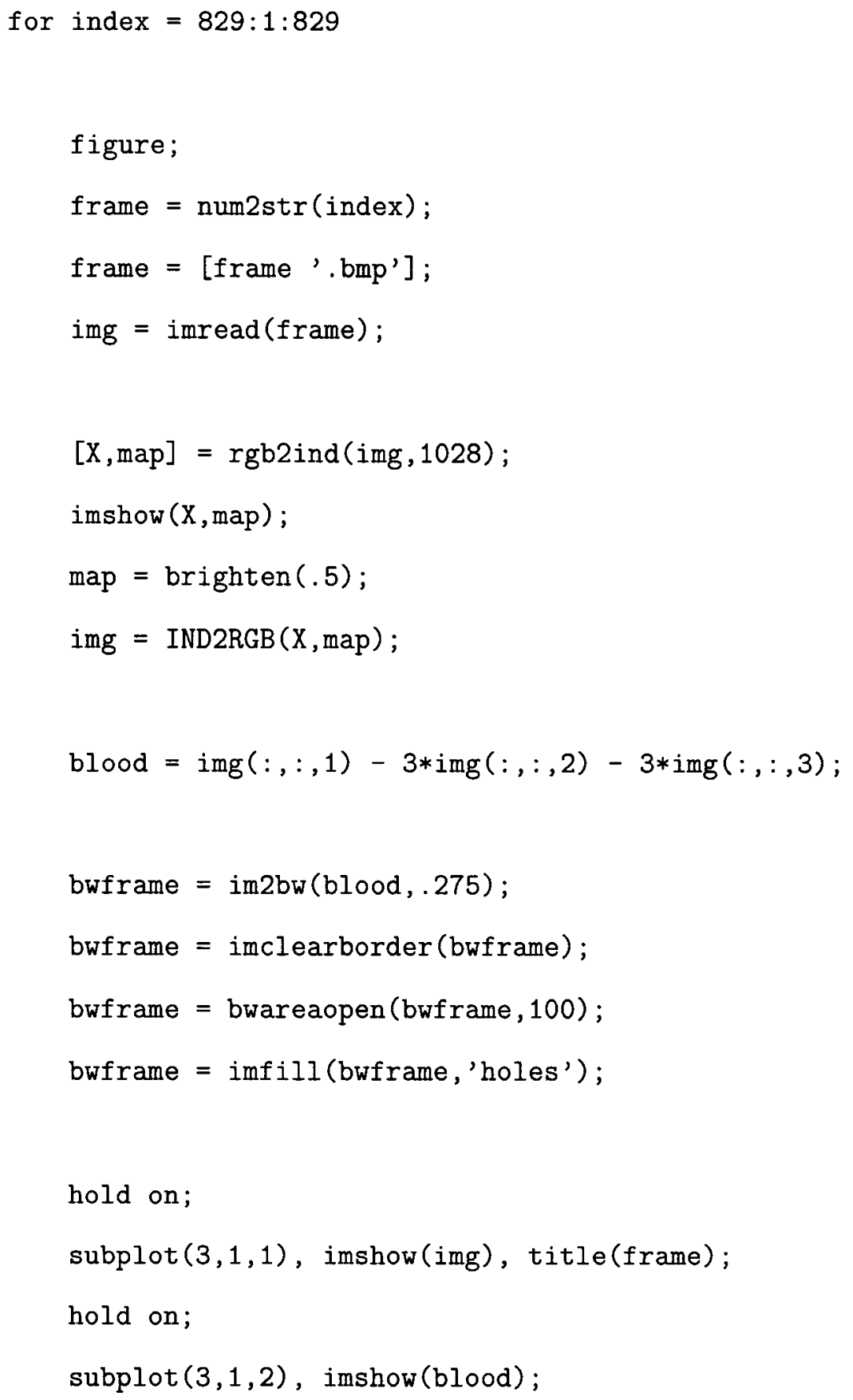




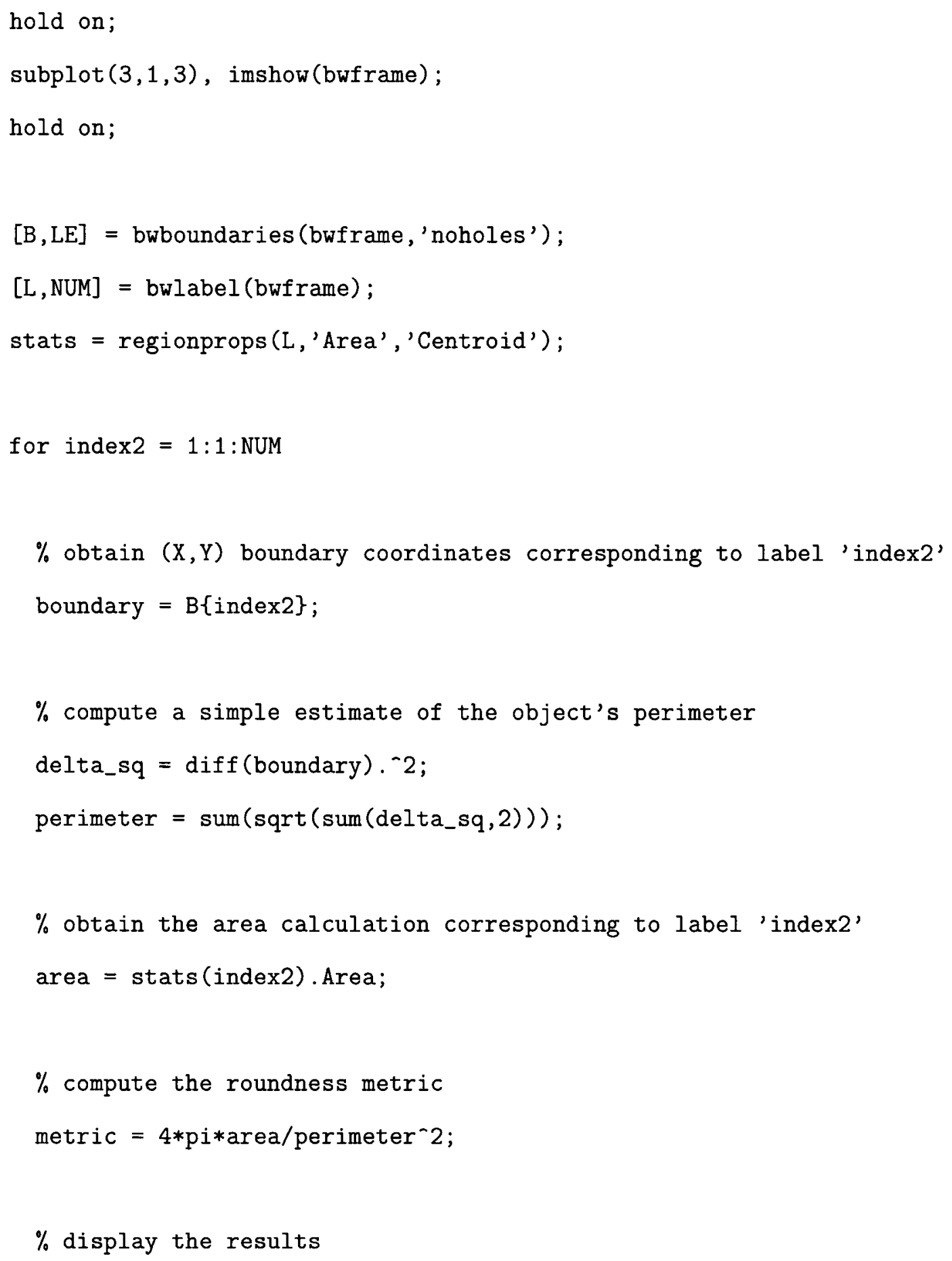




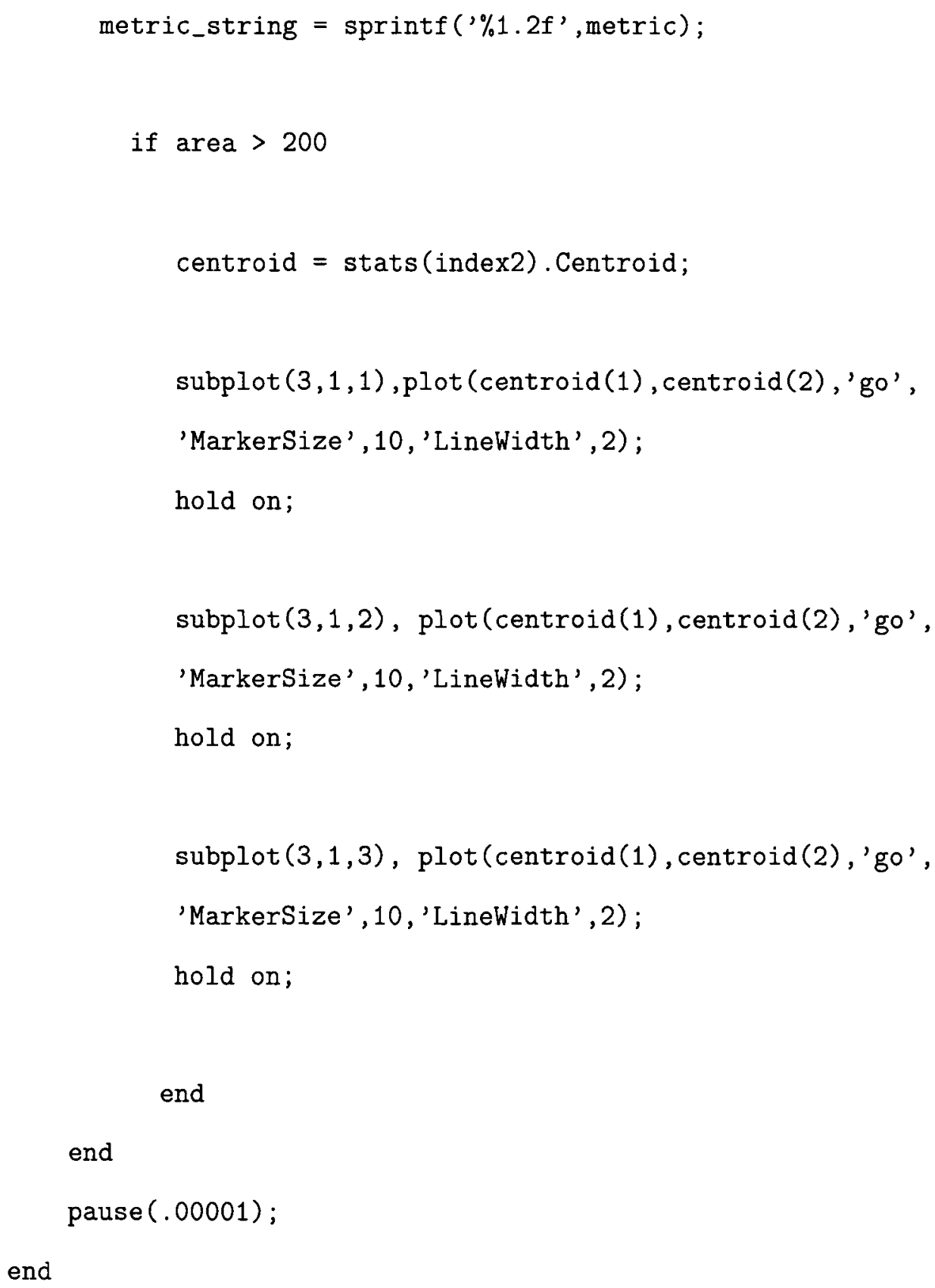




\title{
Appendix B
}

\section{Image Processing Algorithm -}

\author{
Absolute
}


clear;

for index $=829: 1: 829$

figure;

frame $=$ index;

frame $=$ num2str $($ frame $) ;$

frame $=[$ frame '.bmp' $] ;$

$\operatorname{img}(:,:,:$,index $)=\operatorname{imread}($ frame $)$;

$\operatorname{rtr}=15 ;$

$\operatorname{gtr}=4$;

$\operatorname{btr}=4 ;$

$\mathrm{R}=70$;

$G=2$;

$B=2$;

for $i=1: 1: 240$

for $j=1: 1: 320$

$r=\operatorname{double}(\operatorname{img}(i, j, 1$, index $)) ;$

$g=\operatorname{double}(\operatorname{img}(i, j, 2$, index $))$;

$\mathrm{b}=\operatorname{double}(\operatorname{img}(i, j, 3$, index $)) ;$ 


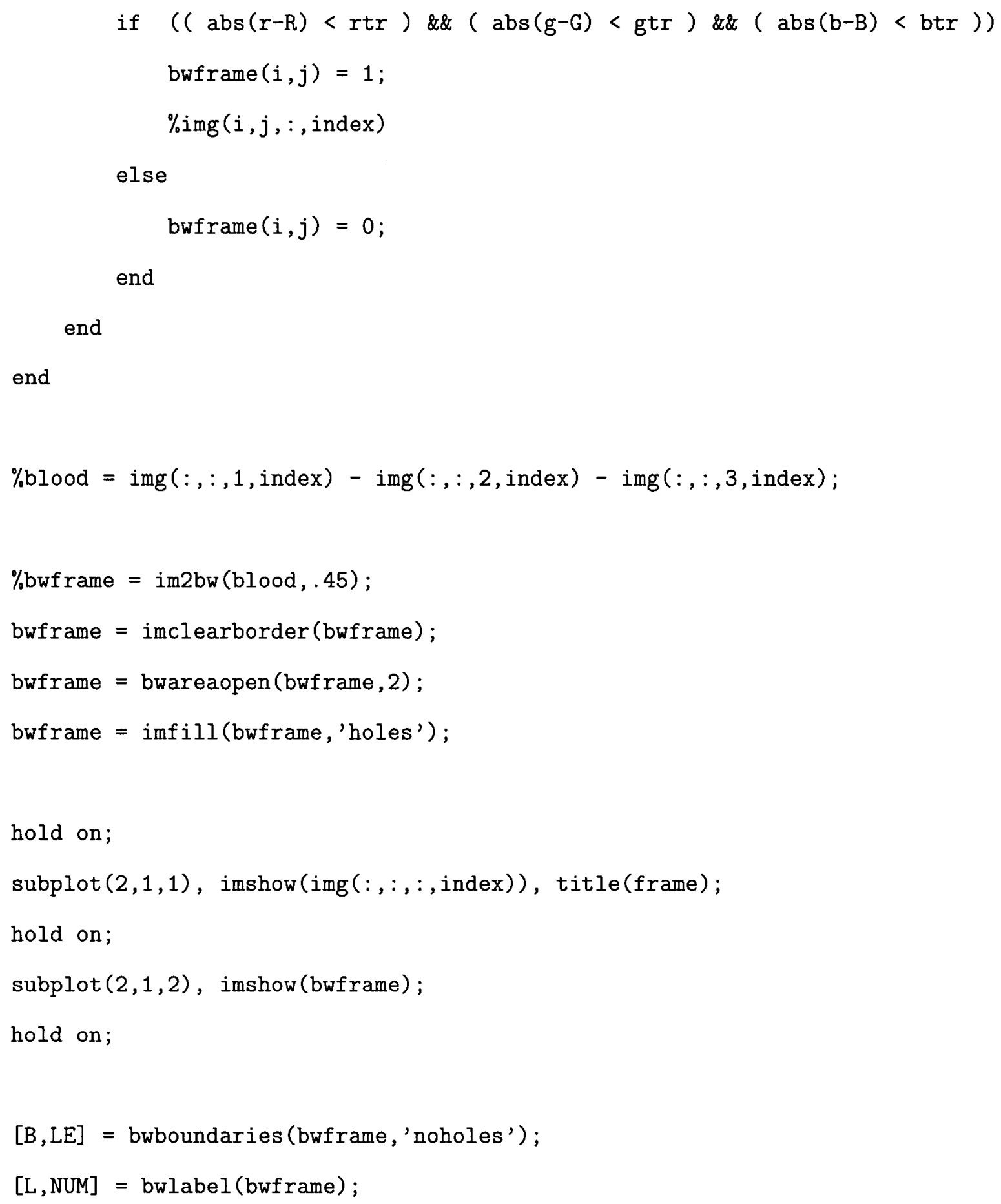




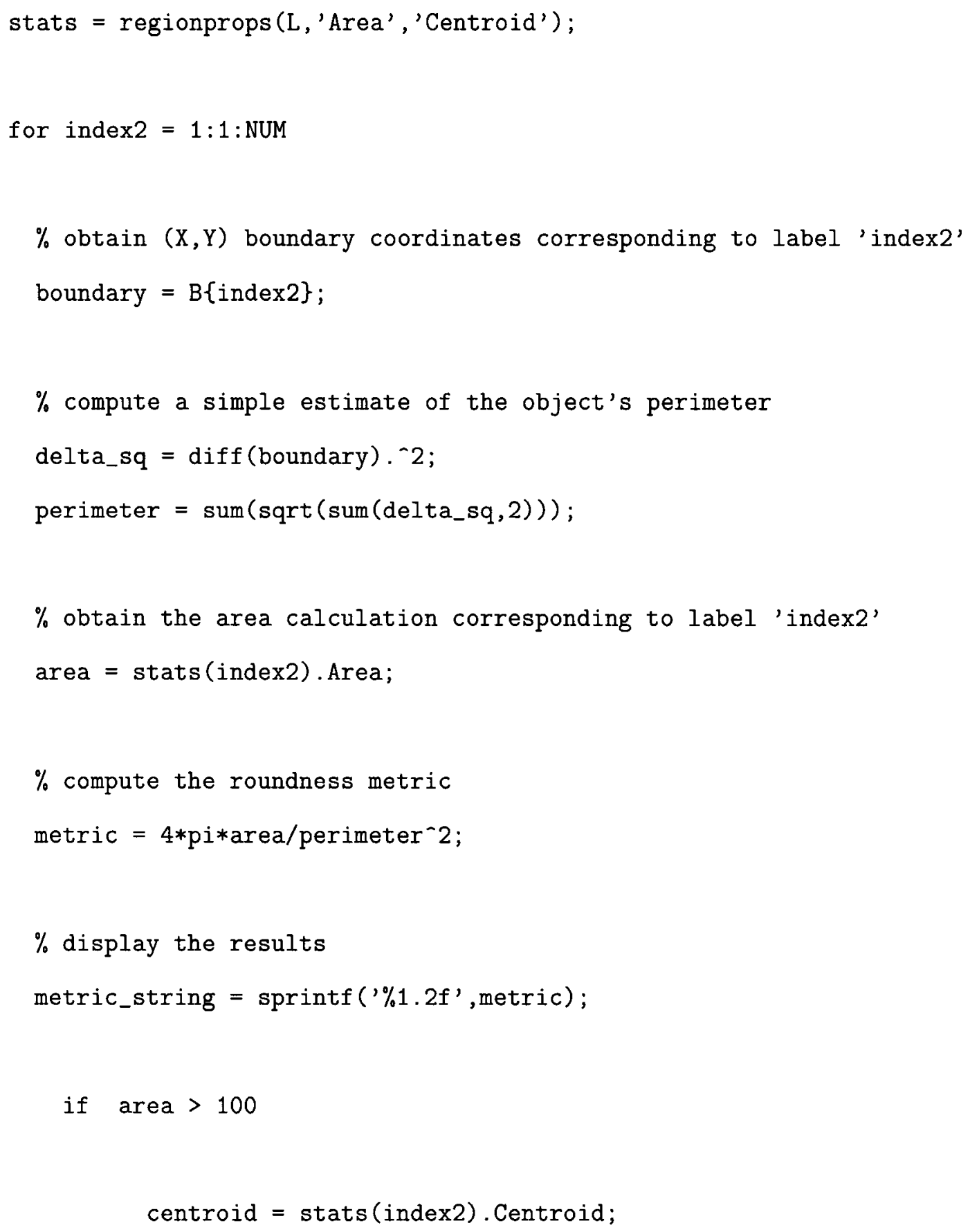




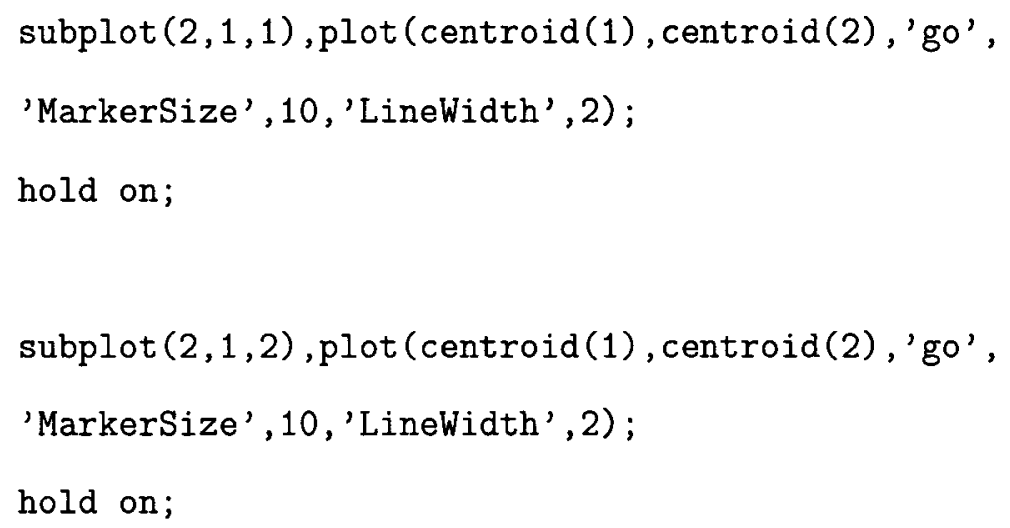




\section{Appendix C}

\section{Design Drawings}




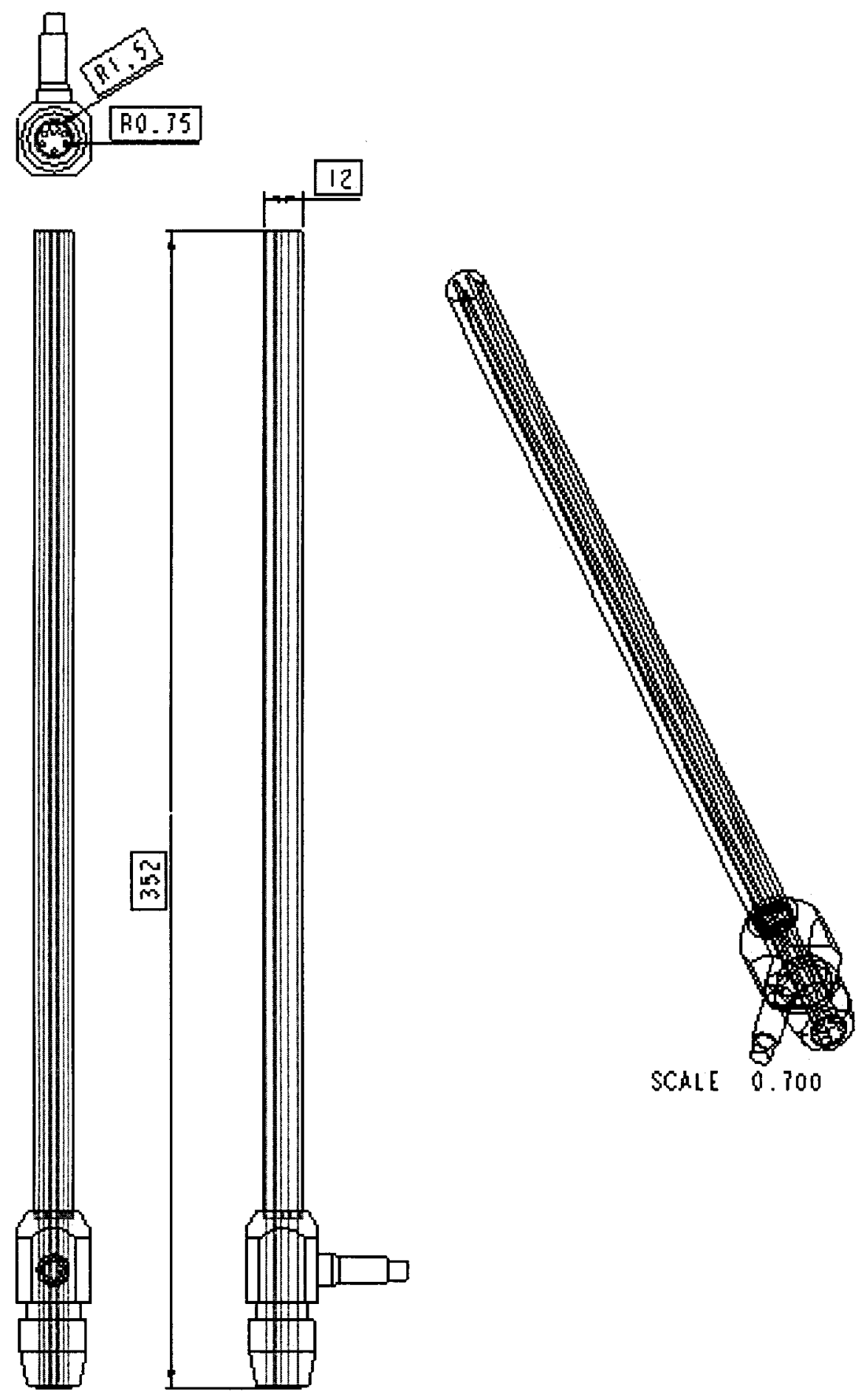

Figure C.1: VACS endoscope design drawing. 

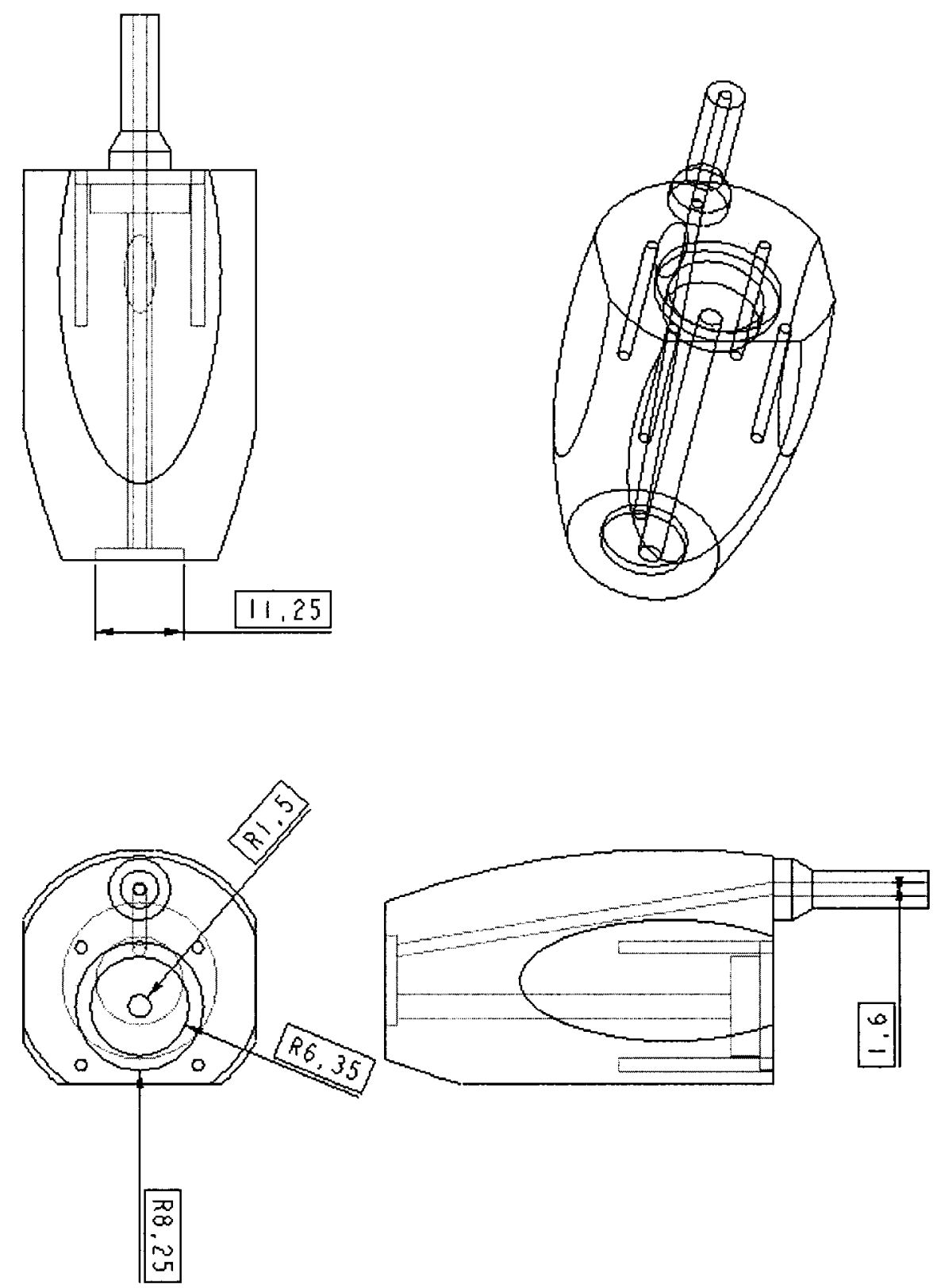

Figure C.2: VACS endoscope adaptor design drawing. 

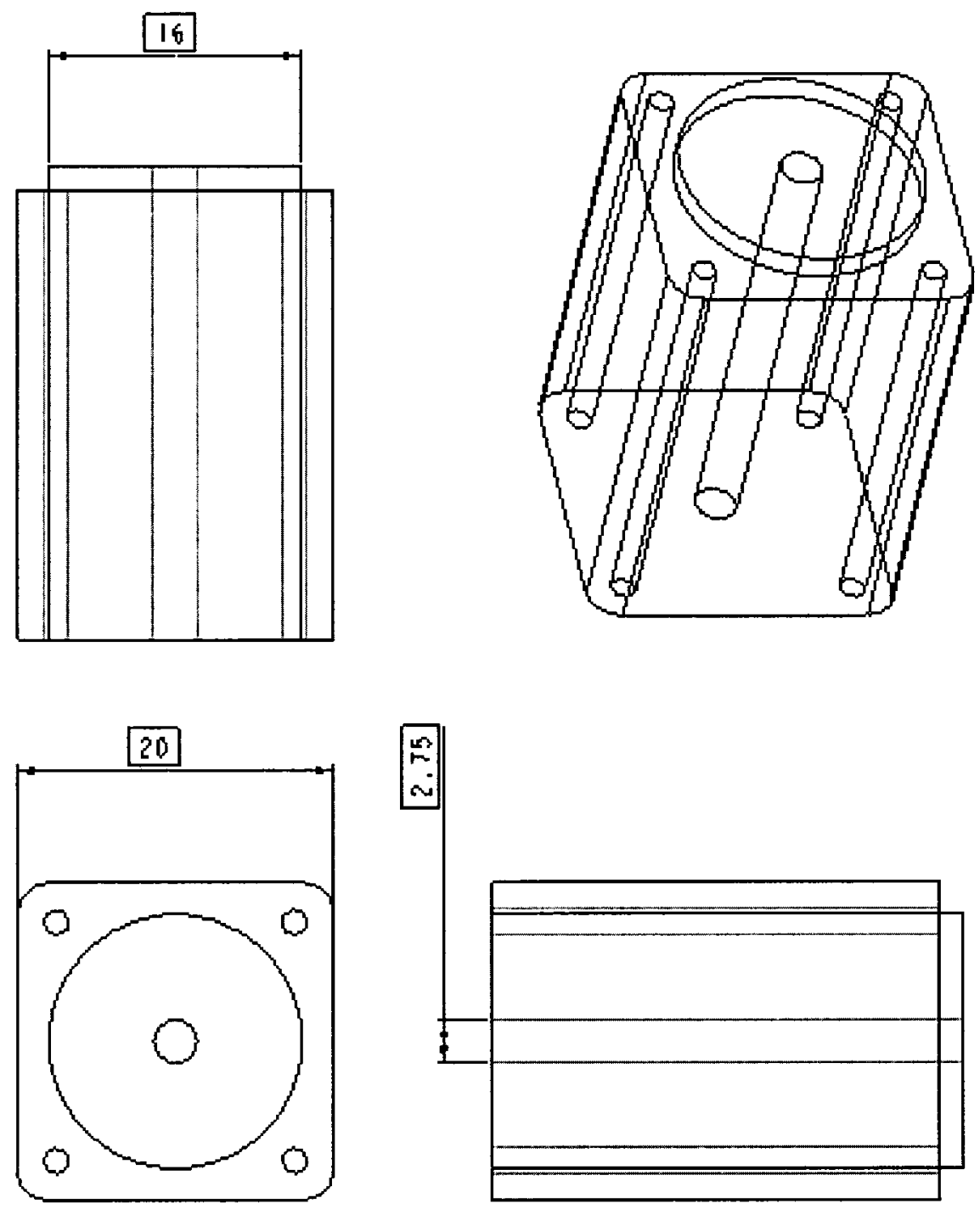

Figure C.3: HSI 21000 Series - Size 8 Non-Captive Actuator Linear design drawing. 

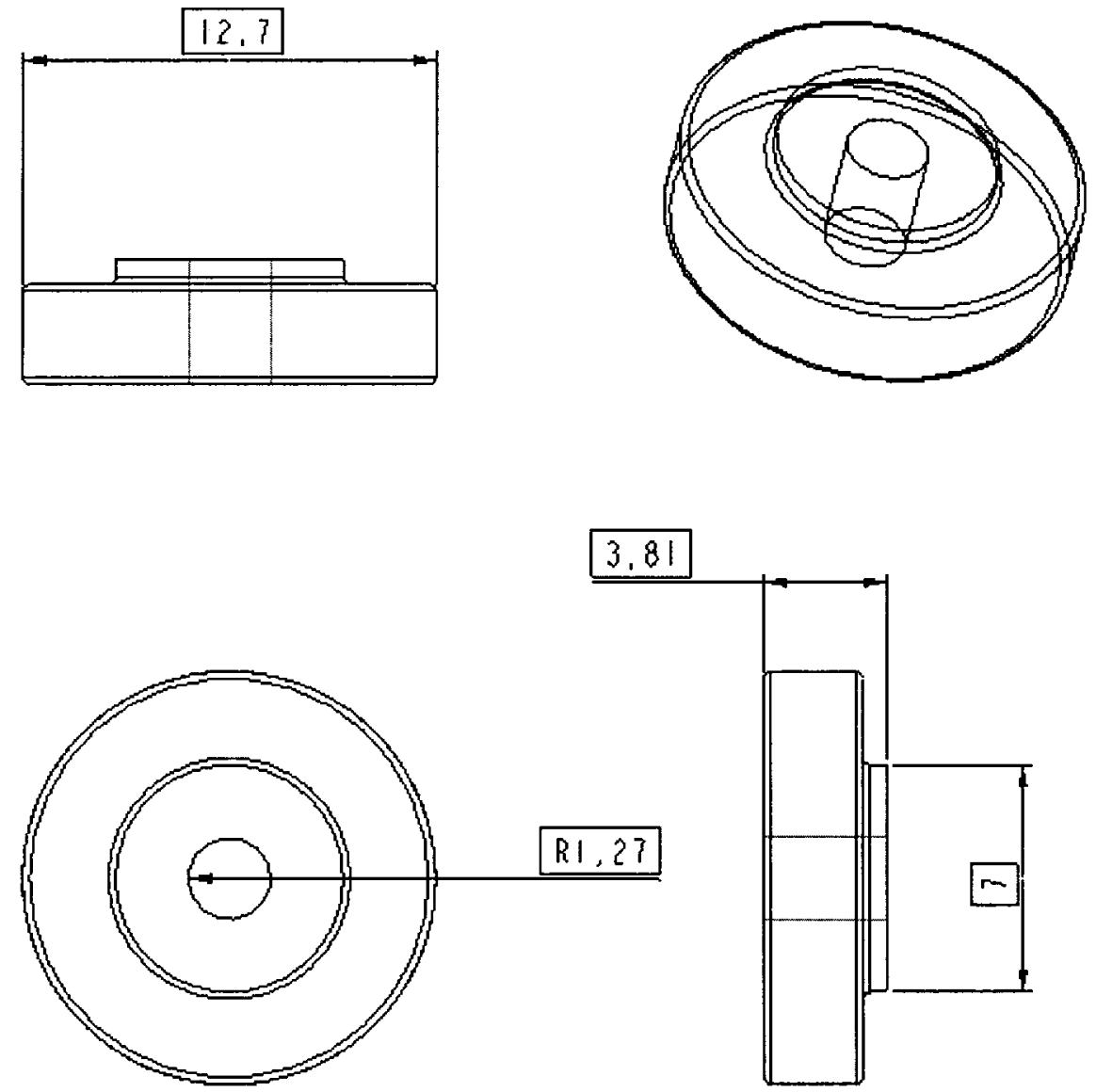

Figure C.4: Honeywell miniature load cell design drawing. 


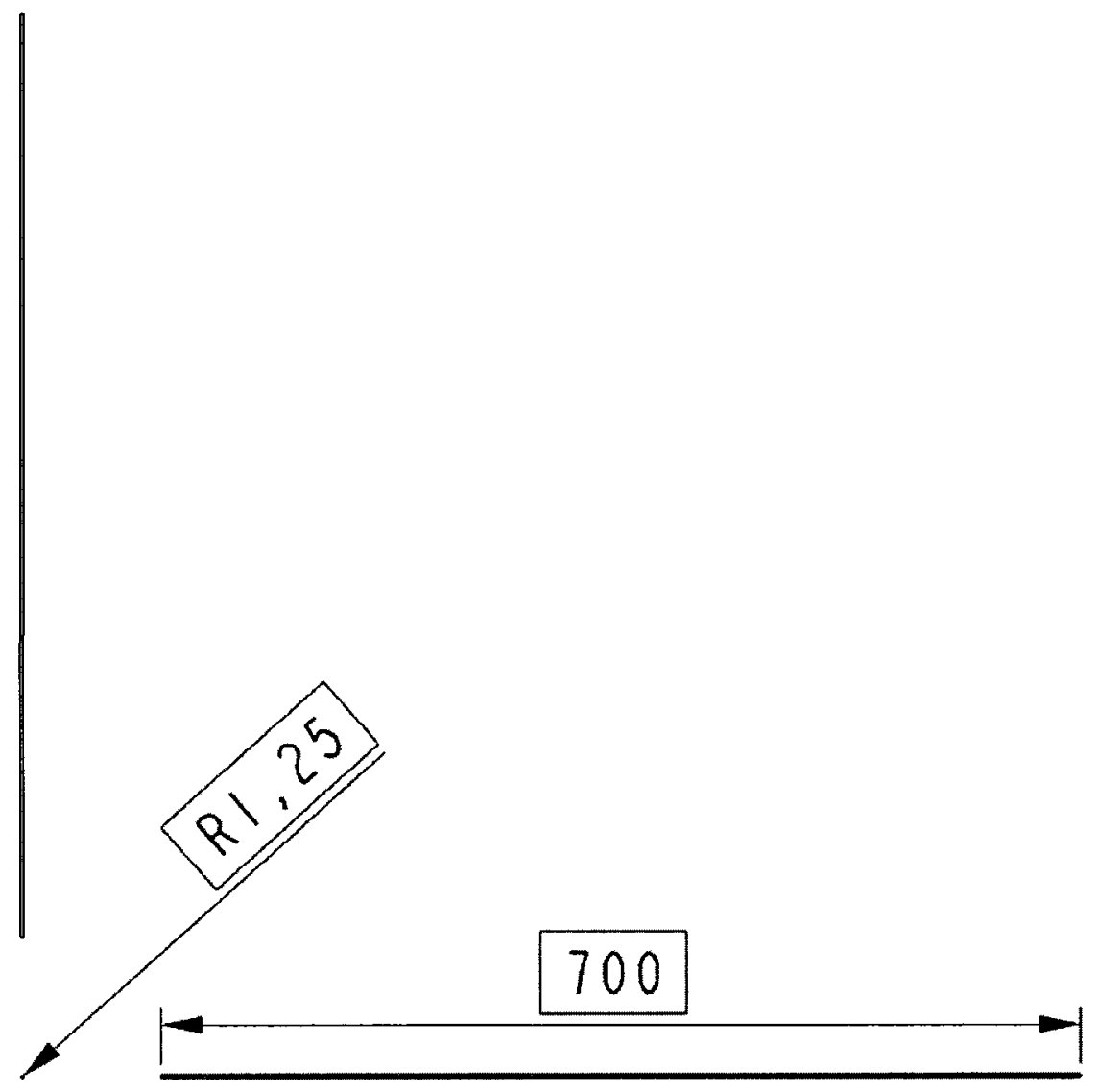

Figure C.5: VACS cautery probe design drawing. 